PALEO

Revue d'archéologie préhistorique

30-1 | 2019

Varia

\title{
Analyse techno-économique d'un assemblage Discoïde du Moustérien récent de l'abri inférieur du Moustier (Dordogne, France)
}

Techno-economic Analysis of a Late Mousterian Discoidal Assemblage from the Lower Shelter of Le Moustier (Dordogne, France)

Marc Thomas et Brad Gravina

\section{OpenEdition}

Journals

Édition électronique

URL : http://journals.openedition.org/paleo/4933

DOI : $10.4000 /$ paleo.4933

ISSN : 2101-0420

Éditeur

SAMRA

Édition imprimée

Date de publication : 30 décembre 2019

Pagination : 300-317

ISSN : 1145-3370

Référence électronique

Marc Thomas et Brad Gravina, «Analyse techno-économique d'un assemblage Discoïde du Moustérien récent de l'abri inférieur du Moustier (Dordogne, France) », PALEO [En ligne], 30-1 | 2019, mis en ligne le 01 juin 2020, consulté le 07 juillet 2020. URL : http://journals.openedition.org/paleo/ 4933 ; DOI : https://doi.org/10.4000/paleo.4933

\section{(c)}

PALEO est mis à disposition selon les termes de la licence Creative Commons Attribution - Pas d'Utilisation Commerciale - Pas de Modification 4.0 International. 

nécessaire pour notre compréhension de la supérieur dans cette région. C'est notamment le cas des industries attribuées au Moustérien de Tradition Acheuléenne (MTA), décrit comme un RÉCENT DE L'ABRI INFÉRIEUR DU MOUSTIER (DORDOGNE, FRANCE) ancêtre potentiel du Châtelperronien. Le site du Moustier, où le Moustérien et le MTA ont été définis pour la première fois, continue de jouer un rôle clé dans ces argumentaires, en particulier à la suite de la révision récente des collections lithiques et fauniques de l'abri inférieur (Gravina et Discamps 2015). Nous présentons ici une analyse techno-économique du matériel lithique provenant de deux unités stratigraphiques identifiées lors des nouvelles fouilles sur le site et corrélables de façon fiable avec la couche $\mathrm{H}$ de Peyrony. Nos résultats à l'abri inférieur du Moustier confirment la réattribution de cette couche au techno-complexe lithique Discoïde et renforcent encore l'impossibilité d'une origine

Marc Thomasa, locale du Châtelperronien.

MOTS-CLÉS Moustérien, technologie lithique, technocomplexe lithique Discoïde, Moustérien de Tradition Acheuléenne de type B, Neandertal. 
Techno-economic Analysis of a Late Mousterian

Discoidal Assemblage from the Lower Shelter

of Le Moustier (Dordogne, France)

Better characterizing the late Mousterian in southwestern France is of particular interest for our understanding of the Middle to Upper Palaeolithic transition in this important region. This is particularly the case for industries assigned to Mousterian of Acheulean Tradition (MTA), which has been described as a potential ancestor of Châtelperronian. The site of Le Moustier where both, the Mousterian and the MTA were first defined continues to play a key role in these arguments, especially following a recent revision of lithic and faunal collections from the lower shelter (Gravina et Discamps 2015). Here we present a technoeconomic analysis of lithic material from two stratigraphic units identified during new excavations at the site and reliably correlatable with Peyrony's layer H. Our results confirm the reattribution of this layer to the Discoid lithic techno-complex and further reinforce the absence of evidence for a local origin of the Châtelperronian at le Moustier.

\section{INTRODUCTION}

De nombreux facteurs, concernant aussi bien la chronologie, les changements de conditions climatiques, la fonction et la réduction des outils en pierre ou encore les mécanismes de transmission culturelle sous-tendent sans aucun doute la variabilité observée dans les ensembles lithiques du Paléolithique moyen. Dès les années 1960, la dissociation de l'influence relative de chacun de ces facteurs a suscité un débat considérable et a conduit, plus récemment, l'archéologie paléolithique à non seulement reconsidérer ses approches méthodologiques systématiques, mais aussi à développer de nouvelles méthodes analytiques, s'éloignant des typologies bordiennes privilégiant une approche technologique qui fait de la taphonomie et de l'intégrité stratigraphique des conditions d'analyses préalables essentielles.

Cette approche renouvelée a conduit à reconnaître que nombre des assemblages lithiques originaux mobilisés par F. Bordes dans les années 1950 pour définir ses « faciès » sont inappropriés pour développer et tester des modèles d'adaptations technologiques, de pratiques de subsistance ou de mobilité des groupes néandertaliens. Cela est dû en partie à la révision critique des assemblages précédemment fouillés, souvent combinée à de nouveaux travaux de terrain qui conduisent à de nouvelles approches quantitatives et qualitatives des séries paléolithiques.

Au premier rang de ces «faciès » typologiques, le Moustérien de Tradition Acheuléenne, ou MTA, est celui qui pose le plus de problèmes. Défini essentiellement sur la base de la présence variable de bifaces, les assemblages initialement attribués à ce "faciès » englobent en réalité des séries dont la variabilité technologique est forte. Par exemple, en s'appuyant sur des travaux antérieurs (Soressi 2002 ; Reubens 2013), mais aussi, en se basant sur un examen complet de contextes archéologiques fiables ou récemment étudiés, on voit que les bifaces ne constituent qu'une composante minoritaire au sein des assemblages (Faivre et al. 2017). De plus, concernant les séries moustériennes à bifaces, seules trois contextes témoignent d'un techno-complexe qui s'appuie majoritairement sur la pratique du façonnage bifacial (Pech de l'Azé I, couche 4, Le Moustier couche G3/G4, et Jonzac, US 7).

La révision des séries ou l'actualisation des données réalisée grâce à de nouvelles fouilles de gisements précédemment assignés aux faciès MTA de Bordes apparaît donc fondamentale pour mieux comprendre l'articulation des technologies bifaciales avec les systèmes de production d'éclats auxquels elles sont souvent associées.

Les travaux de terrain en cours sur le site du Moustier, entamés en 2014, offrent une nouvelle opportunité de tester les récentes réattributions (Gravina et Discamps 2015) d'assemblages anciennement fouillés qui ont servi de base à la définition du MTA (Peyrony 1920) et à sa division ultérieure en types, i.e. A et B (Bordes 1953). Mieux caractériser le Moustérien final au Moustier paraît essentiel à la suite des recherches antérieures plaidant pour une émergence directe du Paléolithique supérieur (c'est-à-dire le Châtelperronien) à partir d'un substrat moustérien local dans le sud-ouest de la France. Nous présentons ici une analyse technologique détaillée d'une 
série lithique récemment mise au jour et soumise à des tests taphonomiques (voir Thomas et al. ce volume) et dont la corrélation avec le sommet de la couche $\mathrm{H}$, définie par D. Peyrony et initialement attribuée au MTA B a pu être établie de manière fiable.

\section{1 | L'ABRI INFÉRIEUR DU MOUSTIER}

\section{1 | Localisation et environnement immédiat}

Le gisement du Moustier est situé sur la commune de Saint-Léon-sur-Vézère (fig.1), au confluent de la Vézère et du Vimont. Composé de trois abris superposés, le gisement du Moustier (Dordogne, France) est un lieu clef dans l'invention et la caractérisation du Moustérien depuis plus de 150 ans (Lartet et Christy 1865-1875; de Mortillet 1869 ; Bordes 1948; Laville et Rigaud 1973 ; Valladas et al. 1986 ; Soressi, 1999, 2002 ; Gravina et Discamps 2015 ; Gravina 2017).

Dans l'environnement immédiat des abris du Moustier se trouve une forte densité de gisements moustériens notamment, les sites dits « classiques » qui ont fait l'objet de fouilles anciennes effectuées par E. Lartet et H. Christy, D. Peyrony ou encore par F. Bordes. Plus récemment, la reprise des activités scientifiques sur les sites dits " classiques » et la fouille de nombreux sites de plein air par l'archéologie préventive ont permis un important renouvellement des connaissances sur le Moustérien du sud-ouest de la France, notamment de ses phases finales (Jaubert 2011 ; Faivre et al. 2017).

\section{2 | Historique de la recherche}

Bien que mentionnées par Lartet et Christy (1865-1875), les premières véritables découvertes à l'abri inférieur sont effectuées par E. Rivière $(1908,1909)$ qui met au jour un crâne ainsi que quelques ossements humains pendant la construction d'une grange dans la partie est du site. L'abri est ensuite fouillé à partir du début du XXe siècle, tout d'abord, par O. Hauser (fouilles en 1909 et 1910) puis par D. Peyrony (fouilles menées de 1912 à 1914). Les principaux résultats des fouilles d'O. Hauser sont les découvertes d'un squelette d'adolescent néandertalien et de quelques ossements isolés. Pour sa part, D. Peyrony met au jour la sépulture d'un enfant néandertalien. De plus, il effectue plusieurs tranchées et un sondage profond (fig. 2) qui lui permettent de proposer pour la première fois une archéoséquence de l'abri inférieur du Moustier. D. Peyrony reconnaît sur une épaisseur de plus de quatre mètres douze niveaux sédimentaires (dénommés de bas en haut par les lettres A à L) parmi lesquels il identifie sept niveaux archéologiques (Peyrony 1930).

En 1948, F. Bordes révise les collections de D. Peyrony et l'archéo-séquence de l'abri inférieur avec comme objectif de caractériser les différences technologiques et typologiques entre le Moustérien typique et le MTA de Peyrony. Selon ses travaux (Bordes 1948), le Moustérien typique se caractérise principalement par un fort pourcentage de racloirs, pas ou peu d'outils à dos et l'absence de bifaces. Selon la vision de Peyrony, le MTA correspond à des industries composées par une faible proportion de racloirs, la présence de quelques éclats et lames à dos et enfin, la présence de bifaces, parfois très nombreux. Selon ces critères typologiques, il attribue les couches B et I de l'abri inférieur au Moustérien typique et les couches F, G et H au MTA. En 1953, F. Bordes apporte une première tentative de re-classification des industries moustériennes. Il distingue cinq principaux faciès dans le sud-ouest de la France : le Charentien, le Moustérien Typique, le MTA de type A (riche en bifaces), le MTA de type $B$ (pauvre en bifaces mais enrichi en couteaux à dos, encoches et denticulés) et le Moustérien à denticulés (Bordes 1953). Suites aux travaux successifs de D. Peyrony (1930), de F. Bordes $(1948,1953)$ et de D. de SonnevilleBordes (1960), la couche $\mathrm{G}$ est attribuée au MTA de type A,

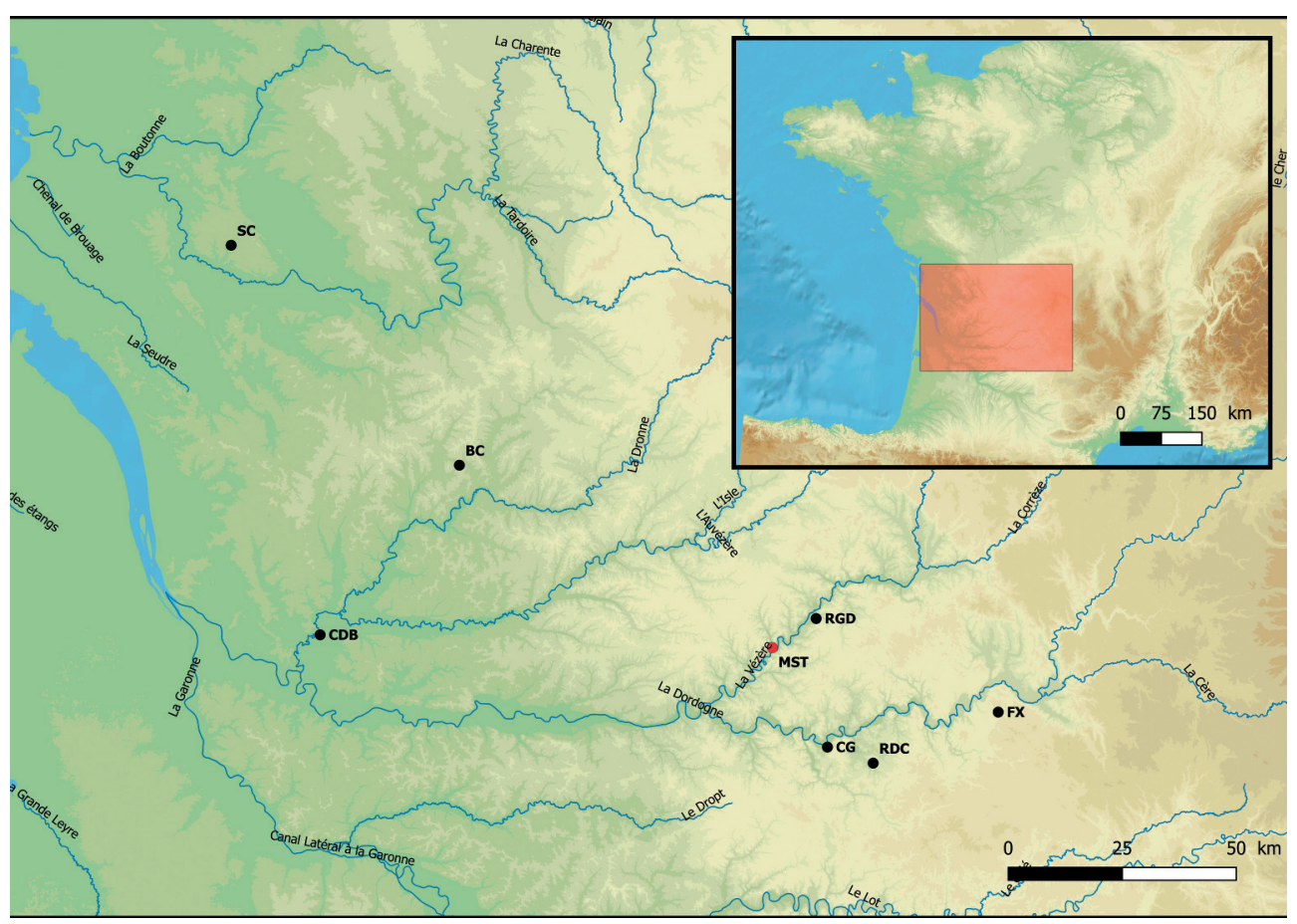

\section{FIGURE 1}

Localisation de l'abri inférieur du Moustier et des sites évoqués dans le texte ( $S C=$ Sainte-Césaire, $\mathrm{BC}=$ Bois Clair, $\mathrm{CDB}=$ Champs de Bossuet, MST = le Moustier, $C G=$ Combe Grenal, $R G D=l e$ Régourdou, RDC = Roc-de-Combe, $\mathrm{FX}=$ les Fieux).

Location of le Moustier and sites mentioned in the text $(S C=$ SainteCésaire, $B C=$ Bois Clair, $C D B=$ Champs de Bossuet, MST $=l e$ Moustier, $C G=$ Combe Grenal, $R G D=$ le Régourdou, $R D C=$ Roc-deCombe, $F X=$ les Fieux)". 


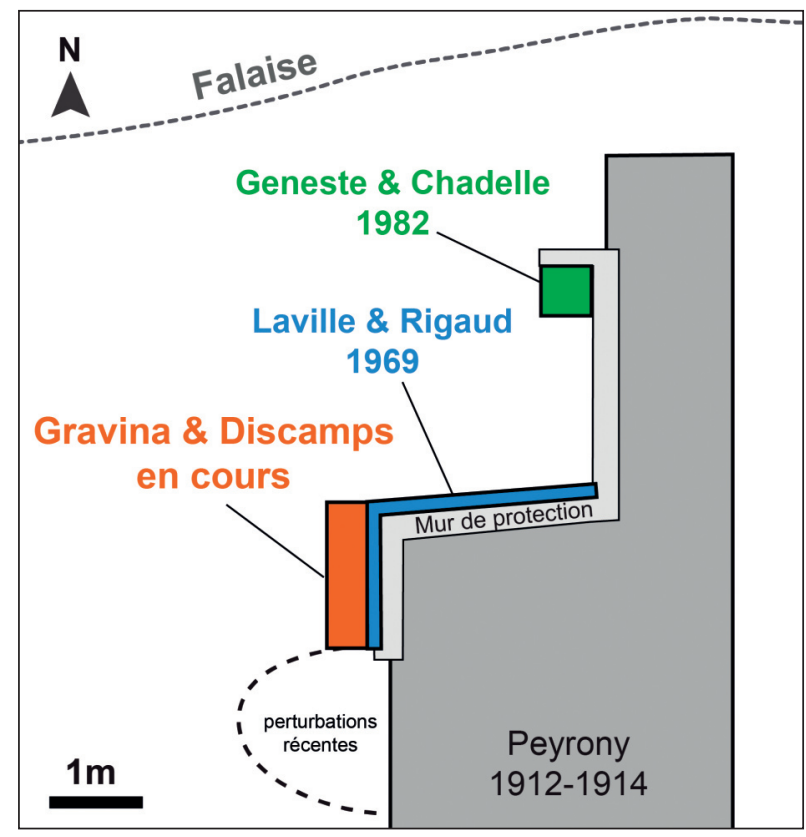

la couche $\mathrm{H}$ au MTA de type B, les couches I et J susjacentes respectivement au Moustérien à denticulés et au Moustérien typique et enfin, les couches $K$ et $L$ à un mélange d'industries aurignaciennes et châtelperroniennes pour la première et à l'Aurignacien pour la dernière. En 1969, H. Laville et J.-P. Rigaud (L\&R) rectifient les coupes de D. Peyrony et précisent la stratigraphie de ce dernier (subdivisions avec une numérotation associée aux couches de D. Peyrony). De plus, ils récoltent un matériel archéologique assez abondant mais ne tamisent pas systématiquement les sédiments. En 1982, J.-M. Geneste et J.-P. Chadelle (G\&C) fouillent environ un quart de $\mathrm{m}^{2}$ (avec tamisage systématique des sédiments) dans le but de récolter des silex brûlés pour des datations absolues par thermoluminescence. Ces dernières situent les dépôts supérieurs de la séquence (couches G à K) entre 56 et 40 ka soit, au début du MIS 3 (Valladas et al. 1986). Deux autres campagnes de datations ont été entreprises par datation ESR (Electron Spin Resonance) et Radiocarbone, respectivement par P. Mellars et R. Grun (1991) et par T. Higham et al. (2014). Celles-ci sont peu ou prou cohérentes avec les résultats obtenus par H. Valladas et collaborateurs et confortent la datation des couches $G$ à $\mathrm{K}$ au début du MIS 3.

Les industries lithiques issues de la couche $G$ de D. Peyrony et les 18 bifaces récoltés dans la couche $\mathrm{H}$ ont été étudiées dans le cadre d'une thèse (Soressi 2002). D'après ces travaux, il y aurait une unité des traditions techniques entre les deux types de MTA (production bifaciale, débitages Levallois, Discoïdes et d'éclats allongés), la différence entre les deux faciès résiderait plutôt dans l'organisation des activités de taille au sein du territoire. Les collections issues de la couche $\mathrm{H}$ fouillée par D. Peyrony (1930) et attribuées au MTA de type B (Bordes 1948, 1953 ; Soressi 2002) n'ont quant à elles pas été revues, à l'exception des bifaces (Soressi 2002 ; Gravina et Discamps 2015), depuis l'étude menée par F. Bordes (1948).

\section{FIGURE 2}

Emprise des différentes opérations de terrain dans l'abriinférieur du Moustier pour les dépôts correspondant aux couches $\mathrm{G}$ et $\mathrm{H}$ de Peyrony. Le rectangle orange correspond à la « Zone B » des nouvelles fouilles.

Location of the different excavations of Peyrony's layers $G$ and $H$ in the lower shelter of Le Moustier. The orange square corresponds to "Zone B» of the new excavations.

\section{3 | La révision des collections J.-M. Geneste}

\section{et J.-P. Chadelle}

La révision des collections issues des fouilles de J.-M. Geneste et J.-P. Chadelle Gravina et Discamps 2015), corrélables à la couche $\mathrm{H}$ de D. Peyrony (Discamps et al. 2016), apportent de nouveaux éléments sur le statut des couches $\mathrm{G}$ et H de D. Peyrony. Dans les niveaux (fouilles $\mathrm{G}$ \& C) corrélables à la couche $\mathrm{H}$ de D. Peyrony, l'un de nous (B.G.) a mis en évidence des industries lithiques dominées par des débitages Discoïdes exclusifs et la présence d'une ramification des chaînes opératoires Discoïdes avec comme objectif la production d'éclats débordants sur la face inférieure d'éclats-matrices. La révision des quelques bifaces issus des fouilles de D. Peyrony présents dans la couche $\mathrm{H}$ montre que quasiment tous portent une doublepatine. Il s'agit probablement d'un recyclage des bifaces ramassés de la couche $\mathrm{G}$ par les occupants de la couche $\mathrm{H}$ pour une production d'éclats à leurs dépens selon une conception Discoïde (Gravina et Discamps 2015). Ainsi, selon la révision des collections issues des fouilles menées par G\&C, l'attribution de la couche $\mathrm{H}$ au MTA de type B (Bordes, 1948 ; 1953 ; Soressi, 2002) est remise en cause au profit du technocomplexe « Discoïde » (Gravina et Discamps 2015), ce concept de débitage étant exclusif dans les sous-niveaux H9 à H2 des fouilles G\&C.

\section{4 | La reprise des fouilles à l'abri inférieur du Moustier}

Les fouilles de l'abri inférieur reprennent en 2014 sous la direction de B. Gravina et E. Discamps (G\&D). Les niveaux archéologiques actuellement fouillés dans la zone B correspondent au sommet de la couche H de D. Peyrony. La base de la couche H n'a pas encore été atteinte. 
Les nouvelles données archéologiques sont recueillies à l'aide de techniques de fouilles modernes (pièces supérieures à $2 \mathrm{~cm}$ coordonnées, tamisage systématique) et soutenues par des approches taphonomiques et géoarchéologiques qui n'ont encore jamais été mises en œuvre à l'abri inférieur du Moustier. Celles-ci permettent la mise au jour de nouvelles collections dont l'intégrité est contrôlée et qui permettent de tester les résultats des études réalisées sur le matériel issu des fouilles anciennes. Ce sont notamment ces approches (voir Thomas et al., Discamps et Lemeur, ce volume) qui ont guidé la définition de trois nouveaux ensembles archéostratigraphiques, numérotés de 1 à 3 du sommet de la séquence à sa base.

\section{2 | CORPUS ET MODALITÉS DE FORMATION DES NAPPES DE VESTIGES}

Le matériel lithique de sept décapages (décapages 6 à 12) issu des fouilles récentes au sein de la zone B correspondant à deux ensembles archéostratigraphiques (1 et 2), a été étudié dans le cadre d'un mémoire de Master (Thomas 2017). La zone B correspond à trois quarts de mètres carrés alignés (C50a, C49c, C49a) qui font l'objet de fouilles depuis 2015. Des décapages de $2 \mathrm{~cm}$ d'épaisseur sont effectués par sous-carré de $50 \mathrm{~cm}$ de côté, tous les vestiges archéologiques de plus de $2 \mathrm{~cm}$ sont cotés à l'aide d'un théodolite et les sédiments issus de chaque décapage sont systématiquement tamisés à l'eau selon des mailles successives de $4 \mathrm{~mm}$ et $1,6 \mathrm{~mm}$.

Sur le terrain, de nouveaux ensembles archéostratigraphiques ont été définis de manière complètement indépendante des découpages précédents. Dans le cadre de cet article, ces ensembles sont temporairement numérotés de 1 à 5 du sommet à la base, en attendant la fin des fouilles dans les différents secteurs et la définition d'un découpage stratigraphique définitif. L'extrême base de la couche $\mathrm{H}$ n'a pas encore été atteinte. Le matériel archéologique est donc redistribué au sein d'ensembles a posteriori lors de la confrontation des observations de terrain aux études du matériel lithique, du matériel faunique (Discamps et Lemeur, ce volume) et suite aux analyses spatiales et taphonomiques (Thomas et al. ce volume). Les deux ensembles considérés ici (temporairement 1 et 2) sont corrélables de façon fiable avec le sommet de la couche $\mathrm{H}$ de la fouille Peyrony (Discamps et al. 2016), datée de la première moitié du MIS 3 (Valladas et al. 1986). Les corrélations avec les subdivisions de la couche $H$ définies par L\&R restent imprécises et largement hypothétiques.

Lors de la fouille, de fortes différences de densité générale en vestiges et de proportions inter-matériaux sont ressenties et ont été définies a posteriori comme ensembles 1 et 2 (fig. 3). Deux grands types de facteurs, susceptibles d'interagir, peuvent être responsables de la répartition spatiale et l'organisation de ces ensembles: le facteur anthropique et le facteur naturel. Pour évaluer l'influence de ce dernier facteur, i.e. l'impact des processus dépositionnels et post-dépositionnels, une étude taphonomique combinée à une analyse de la répartition spatiale des différents types de vestiges est proposée (Thomas et al. ce volume). Dans cet article, seule l'importance du facteur anthropique est testée pour les ensembles 1 (base) et 2 , soit où les différences de proportions entre matériaux sont les plus fortes.

La base de l'ensemble 1, étudiée ici, possède une forte densité en matériel lithique (pour les méthodes de calcul, voir Thomas et al. ce volume). Les industries lithiques issues de cet ensemble se composent d'un total de 1835 pièces cotées. L'ensemble 2 possède, quant à lui, une densité de vestiges lithiques moins importante, il se compose de 863 pièces cotées, soit un total général de 2698 pièces.

\section{1 | Méthode}

Dans un premier temps, le matériel est étudié à l'aide des approches technologiques et économiques classiques, c'est-à-dire vues à travers le prisme de la " chaîne opératoire » (Tixier et al. 1980 ; Geneste 1985 ; Pelegrin et al. 1988 ; Boëda et al. 1990 ; Perlès 1991 ; Boëda 1994). Les débitages Discoïdes sont décrits utilisant les termes et catégories de vestiges employés dans M. Peresani (eds. 2003) notamment par V. Mourre (2003), L. Slimak (2003) et L. Bourguignon et A. Turq (2003). Les catégories ou technotypes employés sont les suivants:

- les « éclats corticaux » sont divisés en trois catégories: les entames dont la face supérieure et le talon sont entièrement corticaux ou néo-corticaux. Sont distingués ensuite les éclats à résidus corticaux ou néocorticaux selon si ces surfaces couvrent plus ou mois de la moitié de la face supérieure de l'éclat en question " éclats corticaux > $50 \%$ » et « éclats corticaux < $50 \%$ »

- les « éclats débordants » emportent une partie du bord du nucléus dans l'axe de débitage. Ils portent un dos abrupt soit cortical ou néocortical, soit formé par un ou des enlèvements antérieurs (fig. 4)

- La dénomination « éclat indifférencié » est utilisée pour des éclats ubiquistes à tous les concepts de débitage du Paléolithique moyen et qui ne montrent aucune récurrence du point de vue de leur forme

- les « éclats centrés courts » n'emportent pas le point de plus forte convexité de la surface de débitage contrairement aux « éclats centrés longs » (Slimak 2003). Le talon est souvent la partie la plus épaisse des éclats centrés courts, souvent plus larges que longs et dont l'axe de l'axe de débitage est strictement centripète

- les «pointes pseudo-Levallois », leur obtention induit un geste particulier, il s'agit d'une percussion selon un axe « ni strictement centripète, ni clairement cordale » (Slimak 2003 : 35) dirigé vers le premier tiers de la surface de débitage. Lorsqu'elle est typique, la pointe pseudoLevallois emporte deux négatifs d'enlèvement orthogonaux à l'axe de débitage qui lui confère un tranchant convergent opposé à un dos déjeté. Suivant le geste décrit précédemment, il est possible d'obtenir des pointes pseudo-Levallois dans de nombreux systèmes de débitage mais, elles ne sont recherchées de manière récurrente que dans le concept de débitage Discoïde stricto sensu (Mourre 2003).

- la seule contrainte inhérente au concept Discoïde se trouve dans l'entretien d'une convexité limitée sur la ou les surface(s) de débitage du nucléus. Plusieurs éclats dits « techniques » peuvent intervenir afin de la réduire: les « crêtes axiales » et les « crêtes frontales » pour ouvrir de nouveaux plans de frappe; les éclats centrés «longs» $(n=3)$ dont l'axe de débitage est strictement centripète 

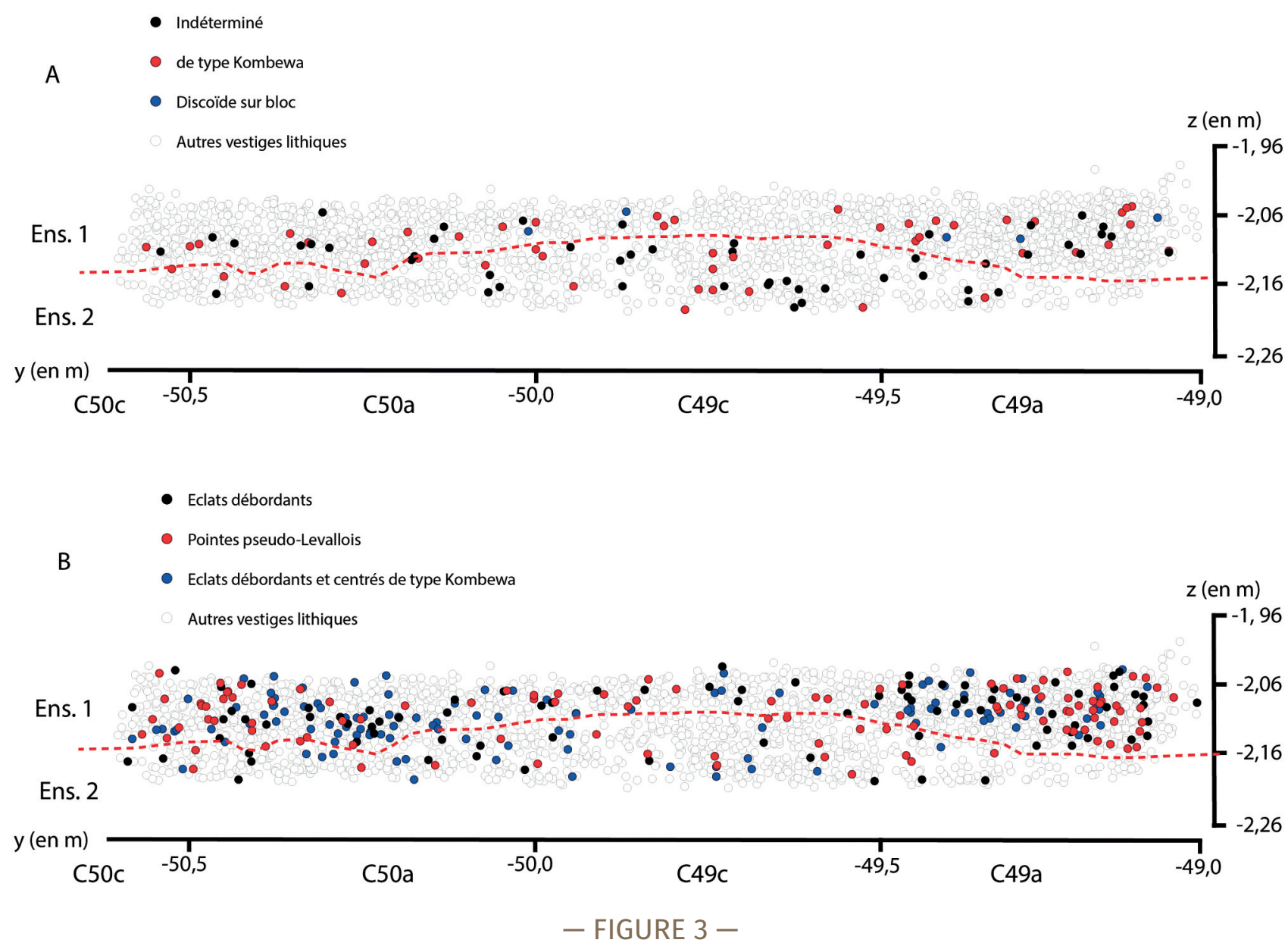

Projection des pièces diagnostics des échantillons étudiés provenant des ensem-

Projection of diagnostic pieces in ensembles 1 and 2. bles 1 et 2 .

(Slimak 2003) et qui emportent le point de plus forte convexité sur le nucléus (ou éclats à « profil brisé » selon Locht et al. 1995)

Sont considérés comme éclats-matrices (fig. 5) tous les éclats sélectionnés afin que soit effectué un débitage dans leur propre épaisseur (Bourguignon et al. 2004). Lorsque la surface de débitage est la face inférieure de l'éclat alors ce débitage est dit « de type Kombewa » (Tixier et al. 1980 . Tixier et Turq 1999 ; Bourguignon et Turq 2003 Bourguignon et al. 2004 ; Faivre 2008). La classification développée par L. Bourguignon et A. Turq (2003) est ici reprise pour l'étude des éclats de type Kombewa. Lors de l'étude des industries lithiques du site de plein air de Champs de Bossuet et de la couche 14 de Combe Grenal, L. Bourguignon et A. Turq (2003) classent les éclats de type Kombewa selon d'une part, le type d'éclat obtenu (éclat de préparation de plan de frappe, pointe pseudo-Levallois, éclat débordant et éclat centré) et d'autre part, selon le nombre d'enlèvements antérieurs visibles sur leur face supérieure. Un éclat de type Kombewa de première génération ne compte aucun négatif d'enlèvement antérieur en face supérieure, un éclat de seconde génération compte un négatif d'enlèvement antérieur et enfin, un éclat de troisième génération en compte deux ou plus.
Les critères de description de la retouche ont été empruntés aux travaux de C. Thiébaut (2005), il s'agit de critères de régularité pour les encoches et les denticulés (type d'enlèvement d'après les travaux de D.-C. Prost, 1989, dimensions, délinéation de la retouche et cohérence de leur position).

Afin de vérifier si les différences de fréquence des types d'objets sont significatives, c'est-à-dire qu'elles ne correspondent pas à des variations aléatoires, des tests du Chi2 (tel que proposé par K. Pearson) sont réalisés sur d'une part : les effectifs de pointes pseudo-Levallois, d'éclats débordants, de supports transformés et, d'autre part, sur les effectifs de nucléus de type Kombewa comparativement au nombre, respectivement de supports entiers et de nucléus. Ces tests sont réalisés sur les différentes collections et niveaux correspondant à la couche $\mathrm{H}$ de D. Peyrony (collections G\&C et ensembles 1 et 2 G\&D). Au sein de ces différents ensembles/us nous émettons l'hypothèse d'une absence de fragmentation différentielle des supports selon leur type et selon les niveaux (voir pour une discussion plus approfondie Thomas et al. ce volume pour les ensembles 1 et 2). De plus, une fragmentation différentielle selon les supports ne pourraient s'expliquer que par une utilisation différentielle de ces derniers (spatialement ou en diachronie) mais difficilement par l'action d'agents taphonomiques, atteignant pour leur part, 

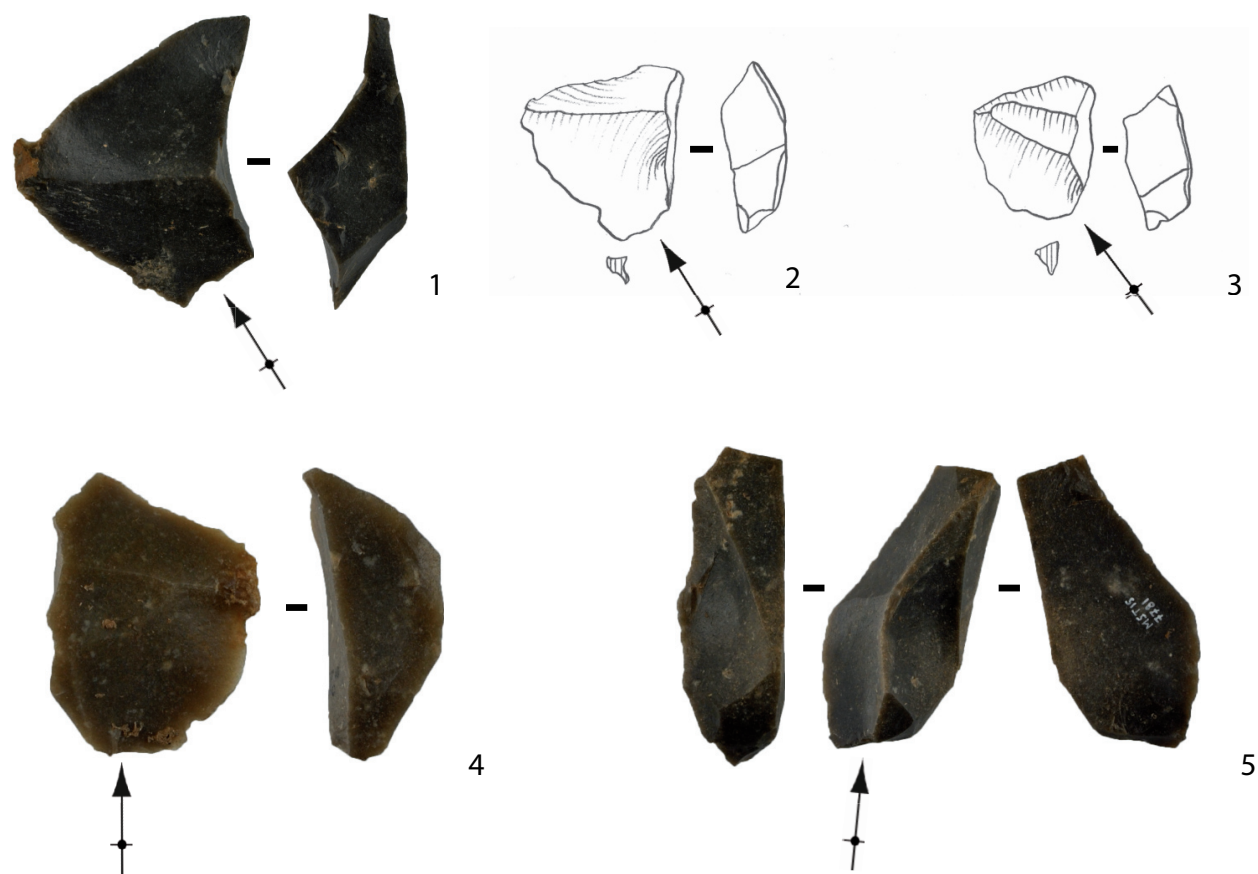

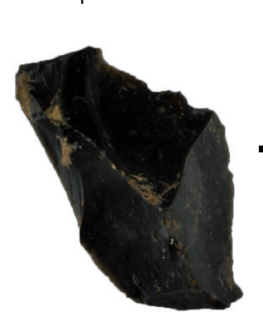

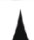

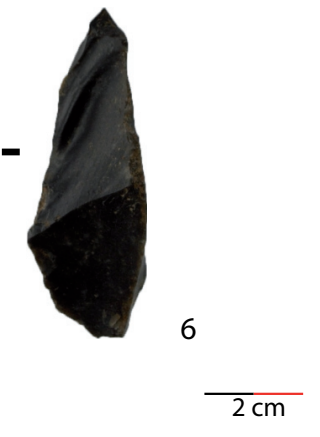

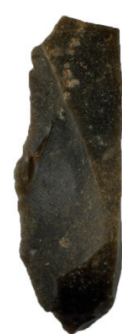
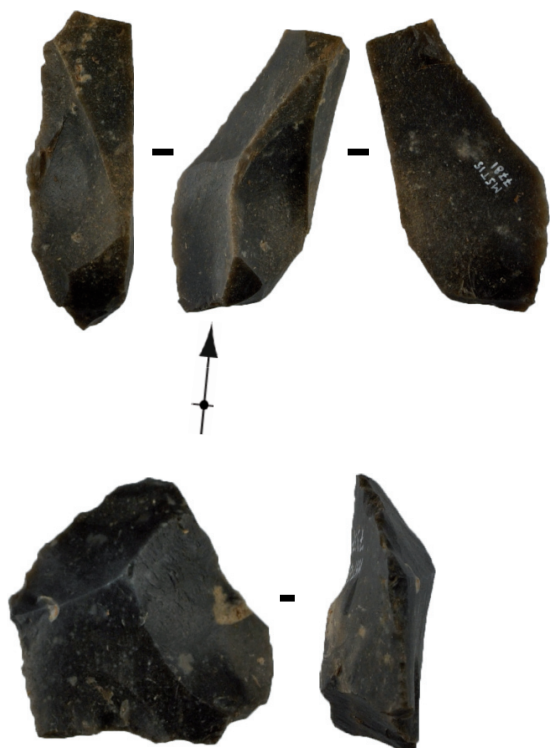

$\Lambda$

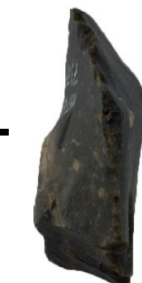

7

\section{FIGURE 4}

Exemples de pièces représentatives des différentes catégories techniques utilisées 1,2,3: Pointes pseudo-Levallois, 4 : éclat débordant dont le dos est formé par un enlèvement antérieur ; 5 : crête axiale ; 6 : éclat centré long ; 7 : éclat centré court.

Examples of diagnostic elements: 1,2,3 : Pseudo-Levallois points, 4: éclat débordant, 5 : axial crest, 6 : long flakes, 7 : short flakes. les supports quel que soit leur type de manière a priori aléatoire. L'homogénéité des critères d'étude du matériel et la définition des types a été assurée par la présence de B. Gravina dans chacune des études réalisées (ensembles 1 et 2, matériel G\&C). En revanche, les différences de fréquence entre certains types de supports et de nucléus entre sites dont les collections n'ont pas été étudiées par les mêmes auteurs peuvent s'expliquer par des critères d'études hétérogènes. Pour cette raison, la comparaison inter-site de la fréquence des types de supports Discoïdes reste strictement qualitative et aucun test statistique n'est réalisé.

Un dernier outil analytique a été mis en place dans le but de juger en premier lieu, de la part de la chaîne opératoire effectuée in situ et, en second lieu, de l'homogénéité des économies mises en place selon les matériaux. Il s'agit du diagramme cumulatif de la répartition des plages résiduelles corticales tel que l'a mis en œuvre A. Tavoso (1978). Le débitage sur galets permet l'observation de plages résiduelles corticales dont la répartition sur la face supérieure et sur le talon des éclats est fonction des modalités de débitages employées et de l'état d'avancement dans la chaîne opératoire. Leur répartition a été classée dans une liste comportant dix-huit rangs (A. Tavoso 1978) qui va de l'entame (E1) jusqu'à l'éclat ne comportant plus aucun résidu cortical (E 18).

\section{2 | Regard critique sur la représentativité des collections Peyrony, G\&C et G\&D}

La collection de la couche $\mathrm{H}$ issue des fouilles D. Peyrony (1930) comprend 332 nucléus, 18 bifaces et «plus de 3000 éclats » (Soressi 2002 : p. 161). Peu de pièces sont inférieures à $3 \mathrm{~cm}$ et la densité de matériel recueilli s'élève à moins de 400 pièces par m3 (Gravina et Discamps 2015). Pour comparaison, le matériel recueilli lors des fouilles G\&D (décapages 1 à 20 de la zone B) a une densité estimée à environ 18000 pièces supérieures à $2 \mathrm{~cm}$ par m3 et pose ainsi question sur la représentativité de la collection D. Peyrony (1930) et ainsi sur la valeur des définitions du MTA.

Les fouilles menées par G\&C se concentrent sur un quart de mètre carré avec tamisage systématique des sédiments mais sans relevé en trois dimensions de la position des vestiges restreignant ainsi les possibilités des études spatiales et taphonomiques (projections spatiales du matériel coté, analyse de la fabrique, analyse de densité). L'étude techno-économique proposée ici menée sur les fouilles actuelles dirigées par G\&D est confortée par les résultats de l'étude taphonomique et spatiale (Thomas et al. ; Discamps et Lemeur, ce volume). En effet, les analyses de la granulométrie des vestiges en silex, l'analyse de leur fabrique, de leur état de surface et la répartition spatiale des liens de raccords effectués mettent en évidence une 

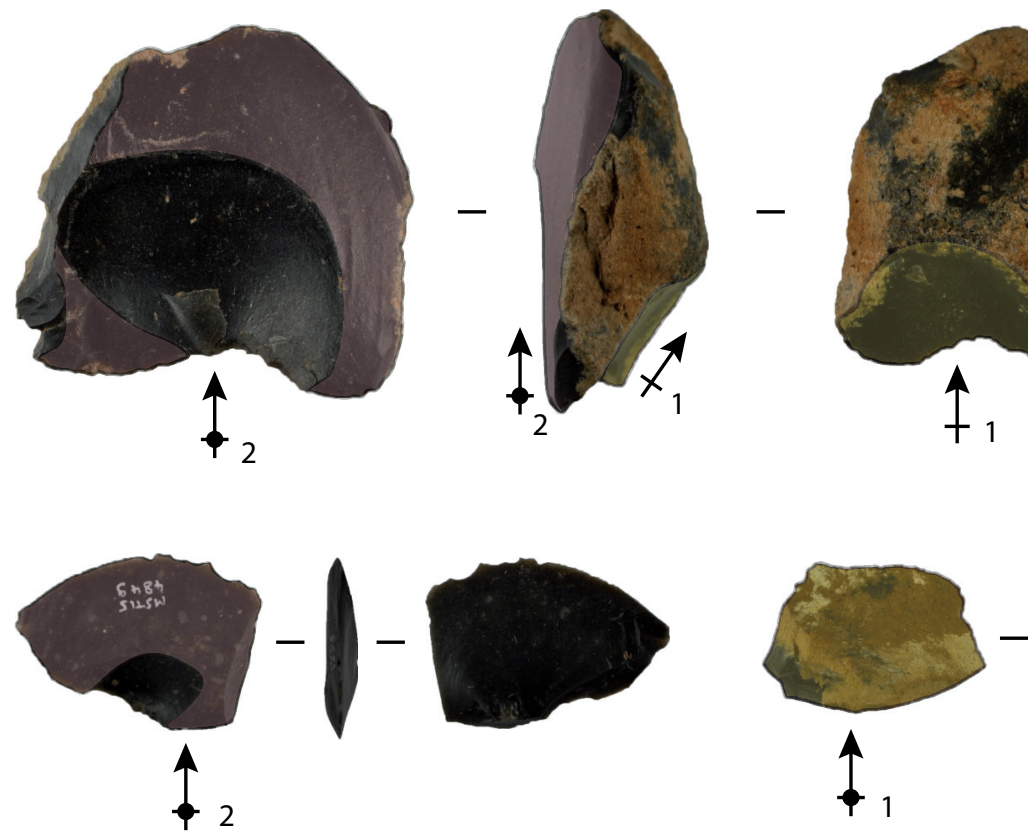

Portion de la face inférieure de l'éclat-matrice

Préparation du plan de frappe

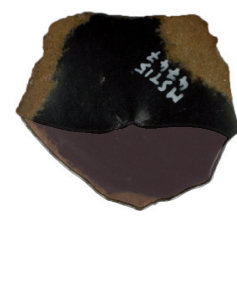

$\overline{2 \mathrm{~cm}}$

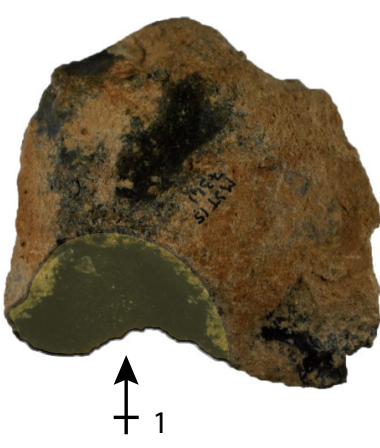

FIGURE 5

Remontage mental d'un débitage de type Kombewa avec préparation d'un plan de frappe (1) et enlèvement de type Kombewa centré (2). La face inférieure de l'éclat-matrice est matérialisée par une couleur rose claire, l'éclat de préparation de plan de frappe et le négatif qu'il laisse par une couleur jaune claire.

Reduction concept for the exploitation of the ventral surface of a flake with the preparation of a striking platform (1) and Kombewatype removal (2). The ventral surface of the reduced blank is in light pink, the striking platform preparation flake andits negative are in light yellow. faible perturbation des industries lithiques des ensembles 1 et 2 qui ne peut remettre en cause leur interprétation en termes technologique et économique.

\section{3 | ÉTUDE TECHNO-ÉCONOMIQUE DU MATÉRIEL LITHIQUE}

\subsection{Composition technique générale du corpus, provenance et modalités d'acquisition des matériaux}

L'ensemble 1 se compose d'un total général de 1835 vestiges lithiques cotés dont 1014 éclats entiers, 58 nucléus, 753 fragments, débris ou cassons et 10 percuteurs ou fragments apparentés. L'ensemble 2 se compose d'un total de 863 vestiges lithiques cotés dont 458 éclats entiers, 48 nucléus, 352 fragments, débris ou cassons et 5 percuteurs ou fragments de percuteurs.

Sur 2698 vestiges, 1867 portent un résidu de cortex alluvial ou du néocortex, soit $69 \%$ du total des vestiges. Cette observation de résidus de cortex roulés ou de néocortex sur la plupart des vestiges quelle que soit la matière première permet d'envisager un approvisionnement qui se concentre quasi exclusivement sur les alluvions. Les alluvions de la Vézère sont aujourd'hui distants d'un peu plus de 200 mètres du site et contiennent tous les matériaux exploités à l'abri inférieur (Turq et al. 2013 ; Turq et al. 2016). Les matières premières importées sur le site (tabl. 1) sont très largement dominées par les silex sénoniens gris à noir (76\% des vestiges de l'assemblage) et blond à brun (22\% des vestiges), suivis par les métaquartzites (moins de $1 \%$ ), la calcédoine (moins de $1 \%$ ), les schistes lités (moins de 1\%) et les silex indéterminés (moins de $1 \%$ ). Les silex sénoniens noirs et blonds présentent une bonne aptitude à la taille. Ces silex sont disponibles au sein d'une large zone géographique (Turq et al. 2013 ; Turq et al. 2016) qui inclue tout le Périgord où ils sont issus des formations géologiques du Crétacé supérieur (Coniacien, Santonien et Campanien). La présence d'entames et de blocs de Sénonien avec seulement quelques enlèvements témoignent d'une importation de matières premières sous forme de galets entiers ou de gros blocs. Les métaquartzites sont peu représentés parmi les artefacts, ils sont issus du Massif central (Colonge et Mourre, 2009) d'où ils sont drainés par les cours d'eau de la Vézère. Seulement quelques vestiges en métaquartzites (mais aucun des vestiges en schistes lités) portent des stigmates de taille. La plupart ont probablement servi comme percuteur. Enfin, la présence de calcédoines dans le corpus n'est que sporadique. Ces matériaux dépendent de formations géologiques tertiaires et leur présence dans les alluvions de la Vézère en amont des abris du Moustier n'est pas à exclure. L'approvisionnement des matériaux est réservé à la sphère strictement locale et se cantonne donc très probablement aux alluvions de la Vézère.

\section{2 | Caractérisation de la chaîne opératoire de production des supports}

Une unique chaîne opératoire de production d'éclats a été identifiée. Il s'agit d'une chaîne opératoire Discoïde (Boëda 1994 ; Bourguignon et Turq 2003 ; Mourre 2003 ; Slimak 2003).

L'objectif au sein de la série semble la réalisation de produits dont la morphologie oppose un dos à un tranchant (tabl.2). Le dos peut être déjeté par rapport à l'axe de débitage dans le cas de la production d'une pointe pseudo-Levallois ( $n=108$, fig. 4 ) ou parallèle à l'axe de débitage dans le cas de la production d'un éclat débordant $(n=87)$. 
Sénonien noir Sénonien blond Sénonien indet Calcédoine Silex indet. Métaquartzites Schistes Total général

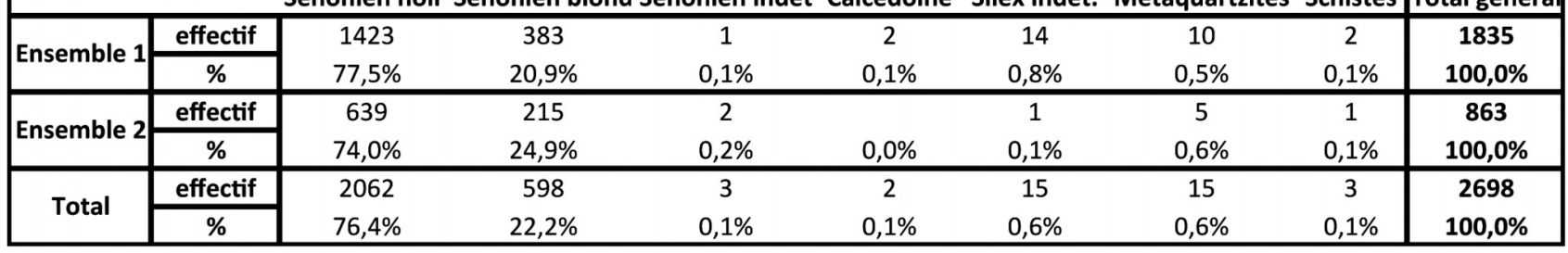

- TABLEAU 1 -

\begin{tabular}{|c|c|c|c|}
\hline & Ensemble 1 & Ensemble 2 & Total \\
\hline Eclats corticaux & 380 & 206 & 586 \\
\hline dont entames & 13 & 12 & 25 \\
\hline dont éclats corticaux $<50 \%$ & 181 & 92 & 273 \\
\hline dont éclats corticaux $>50 \%$ & 186 & 102 & 288 \\
\hline Pointes pseudo-Levallois & 85 & 23 & 108 \\
\hline Éclats débordants & 251 & 92 & 343 \\
\hline dont éclats debordants & 69 & 18 & 87 \\
\hline dont type Kombewa & 67 & 11 & 78 \\
\hline dont dos naturel & 115 & 63 & 178 \\
\hline Éclats centrés & 33 & 17 & $\mathbf{5 0}$ \\
\hline dont courts & 4 & 2 & 6 \\
\hline dont type Kombewa & 29 & 12 & 41 \\
\hline dont longs & 0 & 3 & 3 \\
\hline Crêtes & 5 & 1 & 6 \\
\hline dont axiale & 1 & 0 & 1 \\
\hline dont frontales & 4 & 1 & 5 \\
\hline Éclats de préparation de plan de frappe & 14 & 6 & 20 \\
\hline Éclats indifférenciés & 245 & 113 & 358 \\
\hline Framents (Siret) & $530(36)$ & 253(11) & $783(47)$ \\
\hline Éclat d'encoche & 1 & 0 & 1 \\
\hline Cassons & 46 & 19 & 65 \\
\hline Débris & 177 & 80 & 257 \\
\hline Nucléus & 58 & 48 & 106 \\
\hline dont blocs avec quelques enlèvements & 0 & 2 & 2 \\
\hline dont nucléus Discoïde & 5 & 0 & 5 \\
\hline dont eclat-nucléus type Kombewa & 31 & 19 & 50 \\
\hline dont nucléus indifférenciés (informes et globuleux) & 22 & 27 & 49 \\
\hline Percuteurs et fragments de percuteurs & 10 & $\mathbf{5}$ & 15 \\
\hline Total général & 1835 & 863 & 2698 \\
\hline
\end{tabular}

\section{TABLEAU 2}

Effectifs de vestiges lithiques par catégorie technique.

Lithic remains by technical category.
Le rythme de débitage est continu (Slimak 2003). Chaque éclat est prédéterminé et prédéterminant dans le sens où sa morphologie est recherchée et qu'il possède un rôle d'entretien des convexités sur le nucléus. Le talon des éclats Discoïdes est systématiquement lisse. Lorsque le talon peut être considéré comme dièdre, la percussion est donnée sur l'une des deux faces du dièdre, mais jamais sur la nervure qu'il forme. Les talons ne sont jamais corticaux ni facettés. La technique employée est la percussion directe au percuteur de pierre dure. Cette technique est exclusive tout au long du débitage comme en attestent de forts bulbes et des talons épais.

Le plan de fracturation des enlèvements sur les nucléus Discoïdes n'est pas nécessairement sécant. Le caractère sécant ou non du plan de détachement des enlèvements sur le nucléus rend ainsi compte d'une certaine variabilité (Bourguignon et Turq 2003 ; Mourre 2003 ; Slimak 2003).
La plupart des produits obtenus portent sur leur dos les contre-bulbes d'enlèvements antérieurs réalisés aux dépens de la surface opposée. Ils rentrent ainsi en adéquation avec l'observation de nucléus Discoïdes à deux surfaces de débitage $(n=3)$ dont les surfaces ne sont pas hiérarchisées et peuvent être alternativement surface de plan de frappe et surface de débitage. La gestion du débitage n'est pas systématiquement périphérique, des plages résiduelles corticales restent observables sur les nucléus et sur certains éclats Discoïdes. Deux nucléus Discoïdes ne possèdent qu'une surface de débitage, ils portent sur une surface des négatifs de pointes-pseudoLevallois opposée à une seconde surface majoritairement corticale sur laquelle seulement quelques enlèvements ouvrent des plans de frappe lisses, non-corticaux (fig. 6). 

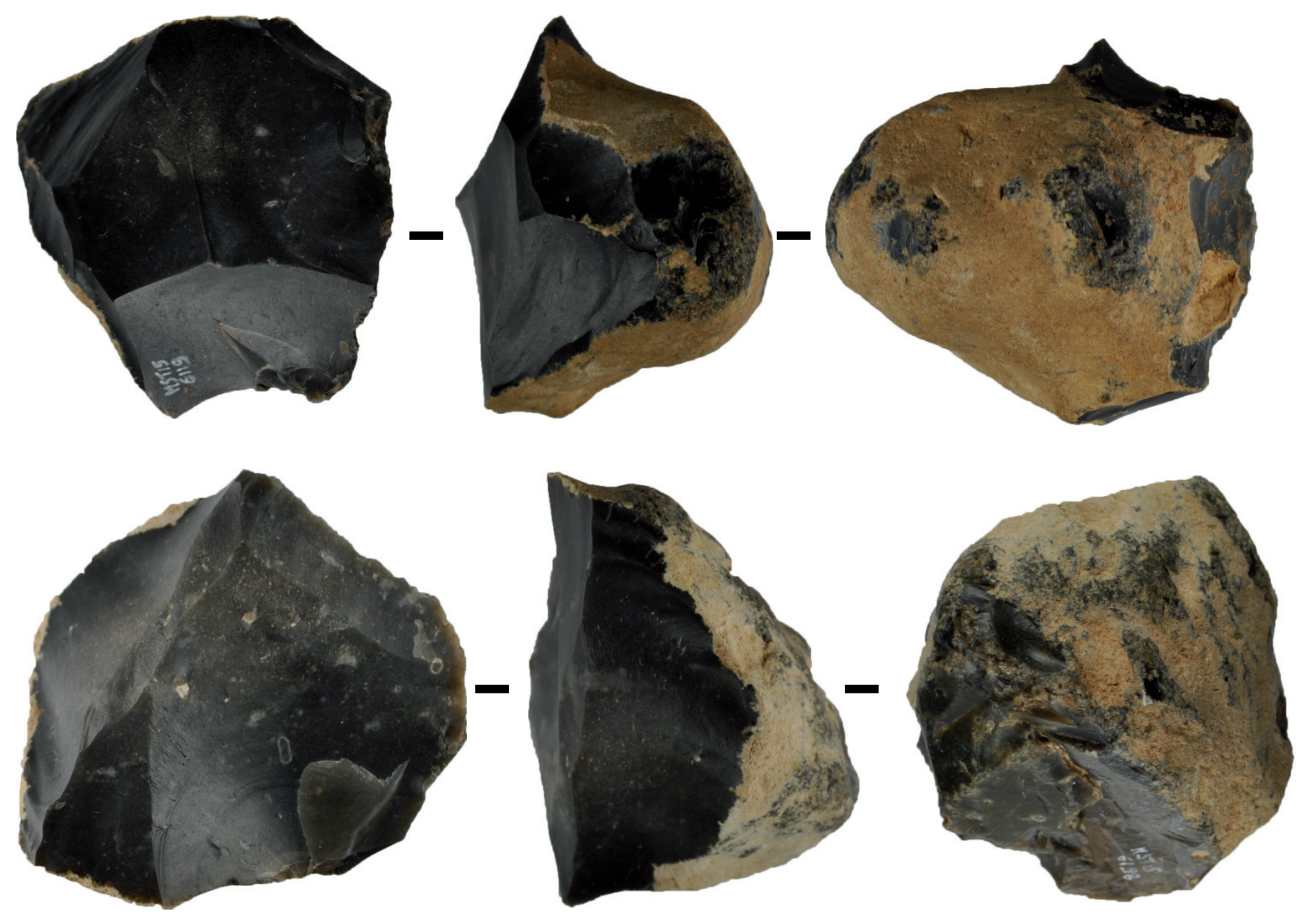

$2 \mathrm{~cm}$

- FIGURE 6 -

Exemples de nucléus Discoïdes unifaciaux issus des ensembles 1 et 2. Un négatif de pointe pseudo-Levallois est visible sur chacun d'entre eux. Malgré la présence d'un résidu néocortical, la surface de plan de frappe n'est pas naturelle car aménagée par des enlèvements de mise en forme antérieurs.

La plupart des nucléus observés sont, à leur stade d'abandon, globuleux et informes. Au sein des séries Discoïdes, l'observation de ces types de nucléus est courante (voir par exemple Locht et al. 1995). La systématisation de la recherche de raccords serait utile afin de mieux les décrire. La production d'éclats indifférenciés selon un enchaînement de gestes plus opportuniste et multidirectionnel en fin de chaîne opératoire ou encore l'irruption d'accidents de taille et de fractures tend à déstructurer les nucléus Discoïdes en fin de chaîne opératoire. Cela a pour effet l'observation de nucléus souvent informes et globuleux à leur stade d'abandon qu'il n'est plus possible de rattacher à une conception Discoïde du débitage. Cependant, eu égard du nombre important de produits Discoïdes dans l'assemblage il est raisonnable de penser que la plupart d'entre eux ont fourni des pointes pseudo-Levallois et des éclats débordants.

\section{3 | Une chaîne opératoire ramifiée}

Lors de la préparation des blocs en amont de la chaîne opératoire Discoïde, les éclats corticaux de plus grandes dimensions peuvent être sélectionnés (fig.7). La largeur et la longueur maximales des éclats corticaux sont en moyenne respectivement de $30 \mathrm{~mm}$ et $41 \mathrm{~mm}$ contre $40 \mathrm{~mm}$ et $53 \mathrm{~mm}$ pour les éclats-matrices. Lorsqu'on compare les dimensions des éclats corticaux et celle des éclats-matrices, le test de Student (Student 1908) donne un résultat significatif pour les comparaisons de chacune
Examples of unifacial discoidal cores from ensembles 1 and 2. A pseudo-Levallois point scar is visible on each example. Despite the presence of neocortex, the striking platform is prepared by previous removals. des deux moyennes, largeur et longueur (respectivement une p-value de 2,7.10-6 et 3,5.10-5). C'est-à-dire, les éclats corticaux de plus grandes dimensions sont sélectionnés et font l'objet d'un débitage mené dans leur propre épaisseur (fig. 8).

L'objectif des débitages sur éclats-matrices est la production d'une part, d'éclats débordants de type Kombewa ( $n=77$ soit $74 \%$ ) et, d'autre part, d'éclats centrés de type Kombewa ( $n=27$ soit $26 \%$ ). Aucune pointe pseudoLevallois de type Kombewa n'a été observée dans l'assemblage (tabl. 3 et 4).

Le détachement de l'éclat de type Kombewa est dépendant de la convexité de la face inférieure de l'éclatmatrice, cela lui confère une «morphologie en éventail » ou plus précisément, un tranchant dont la délinéation est convexe. D'un point de vue strictement qualitatif, nous avons observé que lorsque l'éclat déborde, l'enlèvement est contraint par la convexité de la face inférieure de l'éclat matrice et acquière un angle de tranchant très aigu opposé à un dos. Lorsque l'axe de débitage est centré sur l'éclat-matrice, l'éclat de type Kombewa emporte la convexité de la face inférieure de l'éclat-matrice, cela lui confère un tranchant périphérique relativement fin au profil biconvexe.

Sur les éclats-matrices, des éclats de " préparation de plan de frappe » ouvrent des plans de frappe lisses et dont l'orientation est adéquate au débitage d'éclats de type Kombewa. Les éclats de " préparation de plan de frappe » 


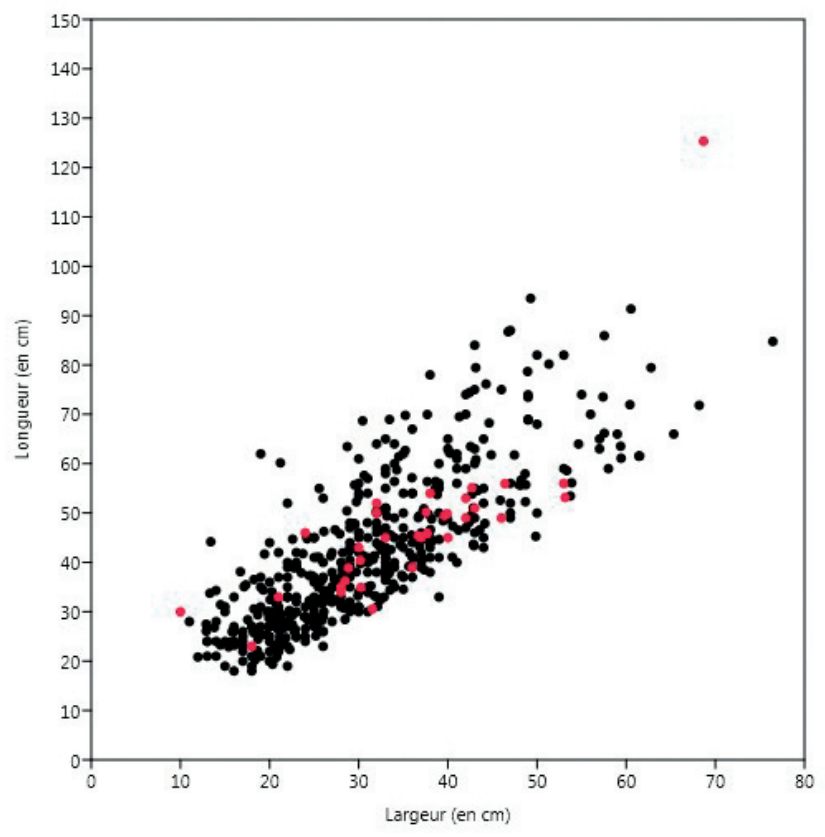

\section{FIGURE 7}

Nuage de point des éclats corticaux par largeur (en abscisse) et longueur (en ordonnées) en mm. Les points noirs matérialisent des éclats corticaux entiers, les étoiles rouges matérialisent les éclats-nucléus portant des résidus corticaux/néocorticaux.

Cortical flakes by width ( $x$-axis) and length (y-axis) in mm. The black dots represent cortical flakes, the red stars represent cores-on-flakes with cortical/ neocortical surfaces.

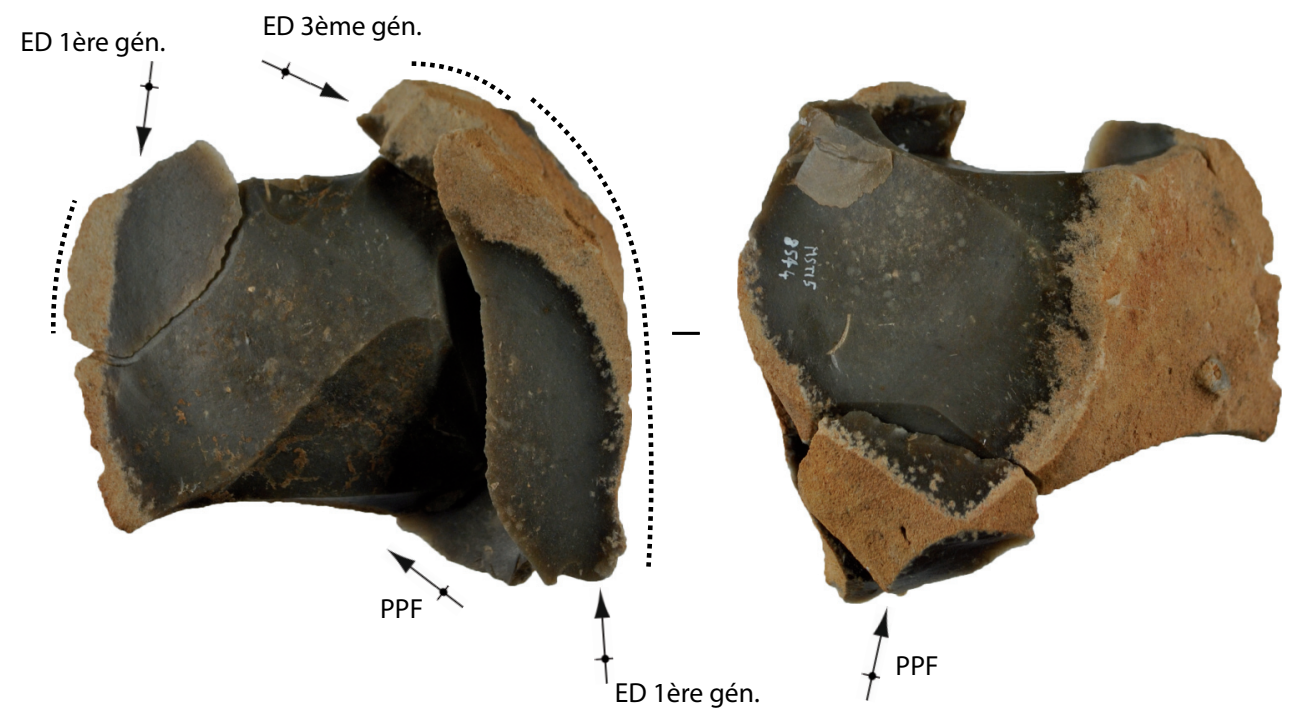

$\therefore \quad$ Dos de l'éclat débordant de type Kombewa

PPF Eclat de préparation de plan de frappe

E 1ère gén. Eclat débordant de première génération

\section{FIGURE 8}

Illustration du remontage d'un débitage de type Kombewa (séquence de débitage longue) de l'ensemble 2.

A refit Kombewa-type reduction sequence (long knapping sequence) from ensemble 2.

\begin{tabular}{|l|ccc|c|}
\hline Type Kombewa (ens. 1 et 2) & 1 ère génération & 2ème génération & 3ème génération & Total \\
\hline Pointe pseudo-Levallois & $0(0 \%)$ & $0(0 \%)$ & $0(0 \%)$ & $0(0 \%)$ \\
Éclats débordants & $43(55,8 \%)$ & $24(31,2 \%)$ & $10(13,0 \%)$ & $77(74 \%)$ \\
Éclats centrés & $8(29,6 \%)$ & $13(48,1 \%)$ & $6(22,2 \%)$ & $27(26 \%)$ \\
\hline Total & $51(49 \%)$ & $37(35,6 \%)$ & $16(15,4 \%)$ & $104(100 \%)$ \\
\hline
\end{tabular}

\section{- TABLEAU 3 -}

Effectifs et pourcentages (entre parenthèses) des types de support de type Kombewa par génération selon la classification de Bourguignon et Turq 2003.
Numbers and percentages (in brackets) of Kombewa-type blanks per generation according to Bouguignon and Turq classification (2003). 


\begin{tabular}{|l|c|c|c|}
\hline Transformation des supports & Ensemble 1 & Ensemble 2 & Total par catégorie \\
\hline couteaux à dos atypique & 14 & 5 & $\mathbf{1 9}$ \\
couteau à dos typique & 1 & 0 & $\mathbf{1}$ \\
denticulés & 4 & 1 & $\mathbf{5}$ \\
encoches & 5 & 1 & $\mathbf{6}$ \\
grattoirs & 1 & 1 & $\mathbf{2}$ \\
racloir simple & 2 & 0 & $\mathbf{2}$ \\
racloir indet. & 0 & 1 & $\mathbf{1}$ \\
fragment indet. & 1 & 1 & $\mathbf{2}$ \\
\hline Total par ensemble & $\mathbf{2 8}$ & $\mathbf{1 0}$ & $\mathbf{3 8}$ \\
\hline Pseudo-outils & $\mathbf{6 2}$ & $\mathbf{1 4}$ & $\mathbf{7 6}$ \\
\hline
\end{tabular}

\section{TABLEAU 4}

Effectifs des supports retouchés par type (Bordes 1961).

Tool types by ensemble (Bordes 1961). portent sur leur talon une portion de la face inférieure de l'éclat-matrice et possèdent une face supérieure majoritairement corticale. Ainsi, le talon des éclats de type Kombewa est toujours lisse, non-cortical et il n'est jamais préparé. La moyenne des angles de chasse des éclats de type Kombewa est en effet de $56^{\circ}$ contre $65^{\circ}$ pour celle des éclats Discoïdes (le résultat du Test de Student est significatif, $p$-value de 1,7.10-7 et avec $t=5,52$ ). L'angle de chasse des éclats de type Kombewa est faible relativement à celui des éclats Discoïdes (hors éclats «techniques »). Cela s'explique aisément par le fait que l'angle de chasse des éclats de type Kombewa correspond à l'angle du bord des éclats-matrices. Le talon est donc incliné.

Pour ce qui est de l'angle d'éclatement, les éclats de types Kombewa obtiennent une valeur moyenne de $110^{\circ}$ contre $108^{\circ}$ pour les éclats Discoïdes (le test de Student est non significatif et renvoie une valeur de $p=0,24$ avec $t=-1,17$ ). Soit, les moyennes des angles d'éclatement des éclats de type Kombewa et des éclats Discoïdes sont équivalentes.

Sur les 37 éclats-matrices qui possèdent deux surfaces hiérarchisées, l'une étant surface de plan de frappe, l'autre surface de débitage, cette dernière ne compte que trois enlèvements maximum dans 32 cas. Douze éclats-matrices présentent également des enlèvements dans leur épaisseur. Comme observé sur les industries lithiques de Champs de Bossuet ou de la couche 14 de Combe Grenal, il s'agit tout d'abord de l'ouverture d'un plan de frappe par une percussion donnée depuis la face inférieure de l'éclatmatrice (Bourguignon et Turq 2003). À partir de ce plan de frappe, un, deux ou trois éclats sont détachés sur la face inférieure de l'éclat-matrice.

Lorsque les chaînes opératoires sont plus longues, la portion de la face inférieure de l'éclat-matrice sur l'éclat de type Kombewa produit à ses dépens se réduit progressivement. Il devient alors difficile voire quasi impossible d'identifier que ces éclats proviennent d'éclats-matrices. Les éclats de première génération sont donc surestimés par rapports aux générations postérieures.

Nous n'avons jamais observé de double patine ni sur les éclats-matrices, ni sur les éclats de type Kombewa. De plus, la proportion des éclats-matrices parmi l'ensemble des nucléus est invariable dans la séquence (cf. infra). Une économie de la matière première est également à exclure puisque l'abri se situe très proche des alluvions de la Vézère qui ont probablement constitué l'essentiel de l'approvisionnement. Ces quelques arguments infirment l'hypothèse du réemploi (réemploi des éclats corticaux disposés sur le sol comme matrices de matières premières par les occupants suivant) mais confirme plutôt l'hypothèse d'un choix délibéré de cette modalité de débitage qui serait ainsi réalisée au début des chaînes opératoires. Enfin, les volumes et les morphologies des galets de silex issus des alluvions de la Vézère offrent des matrices de matière première directement exploitables dans le cadre de débitages Discoïdes. Ainsi, les débitages de type Kombewa seraient motivés par des raisons d'ordre fonctionnel avec comme objectif principal l'obtention de bords à la délinéation convexe dont l'angle de tranchant est très aigu, au profil dont la section est parfois biconvexe.

\section{4 | Activités de transformation des supports}

D'après nos résultats, très peu de supports sont retouchés comme cela est régulièrement observé dans le technocomplexe « Discoïde » (Faivre et al. 2017). Parmi les supports entiers, seulement 2,76 \% et 2,18 \% d'entre eux sont transformés par retouche respectivement pour l'ensemble 1 et l'ensemble 2 (tab. 5 ; fig. 9). Les supports sélectionnés pour être transformés sont soit des éclats corticaux ( $n=24)$, soit des éclats indifférenciés $(n=9)$, dans un cas, un éclat Discoïde centré et, dans deux cas des pointes pseudo-Levallois. Les couteaux à dos sont systématiquement réalisés sur des éclats à résidus corticaux alors que les deux éclats Discoïdes retouchés supportent des racloirs simples. Les encoches et les denticulés, très peu nombreux, sont réalisés sur des éclats à résidus corticaux ou sur des éclats indifférenciés. Ces dernières observations questionnent les intentions et les choix des néandertaliens quant à la sélection des supports transformés par retouche, plus particulièrement pour les couteaux à dos.

En revanche, les pseudo-outils (ici utilisé dans le sens défini par Inizan et al. 1995) sont très nombreux $(n=76)$, plus particulièrement les pseudo-denticulés $(n=19)$ et les pseudo-encoches $(n=49)$. La plupart des supports qui portent des denticulés ont été classés dans les pseudooutils à cause du manque de régularité des enlèvements qu'ils portent (plusieurs types d'enlèvements selon la typologie de D.-C. Prost). Par précaution, lorsque les enlèvements des denticulés et des encoches sont de type $\alpha$ (enlèvement avec fracture conchoïdale, présence d'un contre-bulbe) mais que les supports portent de fortes altérations sur leurs bords ils ont été classés par défaut dans la catégorie des pseudo-outils. Ainsi, nous pensons 


\begin{tabular}{|l|r|r|r|r|}
\hline & H1+H2 & H4-H9 & Ens. 1 & Ens. 2 \\
\hline \% PPL $^{\mathbf{1}}$ & 9,15 & 4,71 & 8,38 & 5,02 \\
\hline \% ED $^{2}$ & 5,37 & 8,61 & 13,41 & 6,33 \\
\hline \% RT $^{\mathbf{3}}$ & 3,35 & 2,80 & 2,76 & 2,18 \\
\hline Effectif total supports $^{2}$ & 2087 & 1359 & 1014 & 458 \\
\hline \% nuc. Type Komb. $^{4}$ & 48,25 & 54,76 & 53,45 & 37,50 \\
\hline Effectif total nuc. $^{\mathbf{}}$ & 114 & 42 & 58 & 48 \\
\hline
\end{tabular}

\section{TABLEAU 5}

Pourcentage des types de supports et de nucléus caractéristiques par ensemble. 1 : Pointes pseudoLevallois ; 2 : Éclats débordants ; 3 : Support retouché ; 4 : Nucléus de type Kombewa ; 5 Effectif total de nucléus.

Percentage of blank types and diagnostic cores per ensemble . 1: Pseudo-Levallois points ; 2 : éclat débordant ; 3 : retouched blank ; 4 : Kombewa-type core ; 5 Total number of cores.
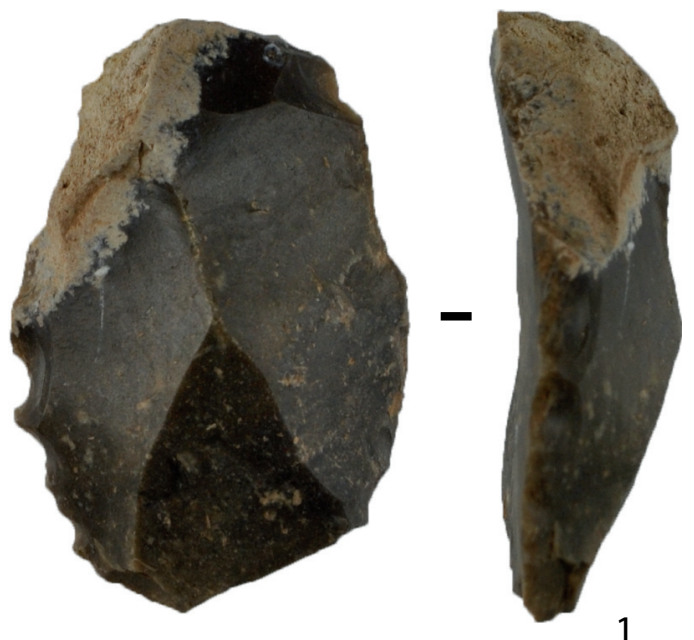
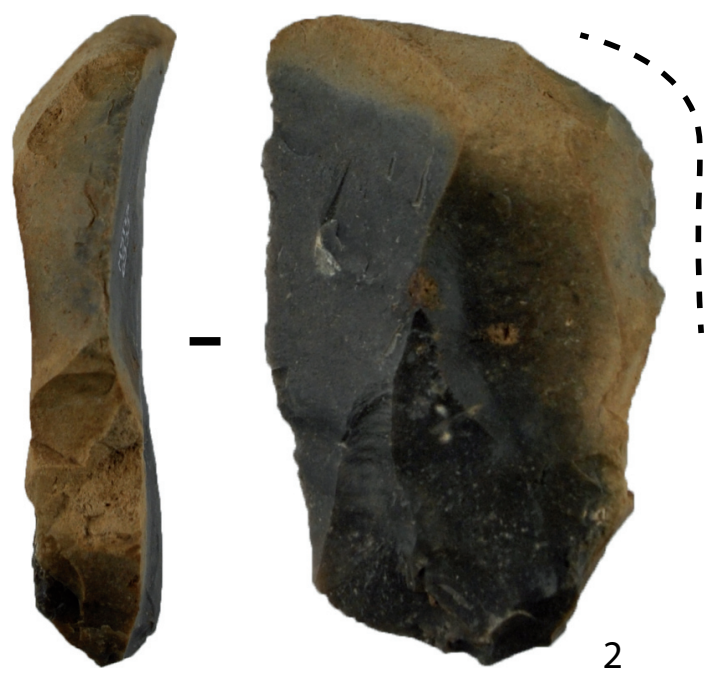

$2 \mathrm{~cm}$

- FIGURE 9 -

Supports aménagés par retouche : 1 : denticulé ; 2 : Couteau à dos atypique.

que nos résultats sous-estiment le véritable nombre d'encoches et de denticulés dans l'assemblage. Malgré cet écueil, notre étude montre une proportion de supports transformés très faible (Faivre et al. 2017), notamment concernant les pièces encochées et les denticulés.

\section{5 | Une gestion des matières premières indifférenciée}

Le diagramme cumulatif met en évidence une très forte homogénéité de la répartition des résidus corticaux ou néo-corticaux quel que soit le matériau (fig.10) renforçant l'argument selon lequel les matières premières font preuve d'une gestion indifférenciée lors du débitage et au sein des économies de production.

\section{4 | TEST D'HOMOGÉNÉITÉ DES ÉCHANTILLONS DE VESTIGES LITHIQUES CORRESPONDANTS À LA COUCHE H DE D. PEYRONY (G \& C, ENSEMBLES 1 ET 2 G \& D)}

Afin de tester l'homogénéité des niveaux concernés au regard de critères technologiques, des tests du $\mathrm{Chi}^{2}$ ont été réalisés deux à deux sur les effectifs des vestiges lithiques diagnostics entre les ensembles archéo-strati- graphiques des fouilles G\&C et G\&D. Ces tests ont été effectués sur les catégories de vestiges diagnostics (nombre de nucléus de type Kombewa sur le nombre de nucléus total) et a priori recherchés (nombre de pointes pseudo-Levallois, d'éclats débordants et de supports retouchés sur l'ensemble des supports entiers). Afin de disposer d'effectifs plus conséquents, les subdivisions respectivement de la base $\left(\mathrm{H}_{1}+\mathrm{H}_{2}\right)$ et du sommet de la couche $\mathrm{H}(\mathrm{H} 4-\mathrm{H} 9)$ sont combinées.

D'après ces tests (tabl.6), quel que soit l'échantillon pris en considération, la couche $\mathrm{H}$ est totalement homogène $\mathrm{du}$ point de vue de la composition des assemblages en nucléus de type Kombewa et de supports aménagés par retouche. En revanche, il apparaît que la quantité de pointes pseudoLevallois et d'éclats débordants n'est pas constante au sein de la couche $\mathrm{H}$. Trois principales hypothèses peuvent être mises en avant : premièrement, les chaînes opératoires Discoïdes admettent une certaine flexibilité de telle sorte que selon les objectifs du tailleur, celui-ci peut produire plus de pointes pseudo-Levallois ou a contrario, plus d'éclats débordants, deuxièmement des éclats débordants et/ou des pointes pseudo-Levallois ont pu être sélectionnés et exportés (et/ou déplacés ou produits à différents endroits sur le site), enfin, ces différences pourraient également s'expliquer par le fait que $\mathrm{H} 1+\mathrm{H} 2$ et $\mathrm{H} 4-9$ ne sont pas strictement corrélables aux ensembles 1 et 2. 

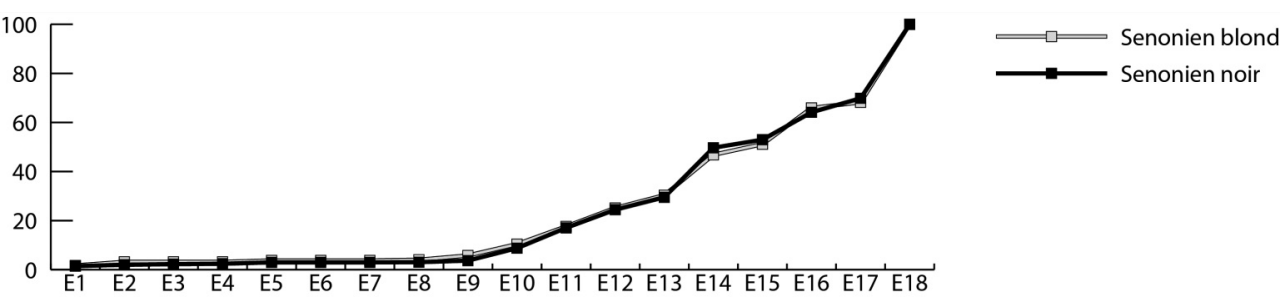

TABLEAU 6
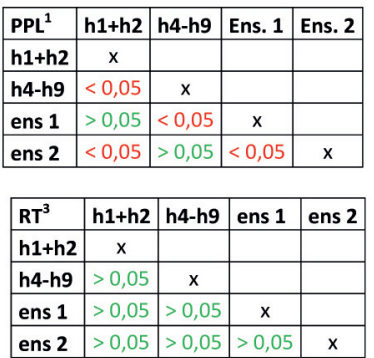

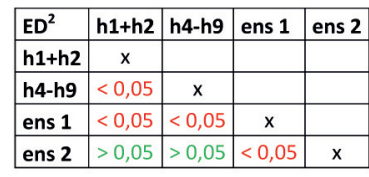

\begin{tabular}{|l|c|c|c|c|}
\hline Nuc. Type Komb. & h1+h2 & h4-h9 & ens 1 & ens 2 \\
\hline h1+h2 & $\mathbf{x}$ & & & \\
\hline h4-h9 & $>0,05$ & $\mathbf{x}$ & & \\
\hline ens 1 & $>0,05$ & $>0,05$ & $\mathrm{x}$ & \\
\hline ens 2 & $>0,05$ & $>0,05$ & $>0,05$ & $\mathbf{x}$ \\
\hline
\end{tabular}

tests du Chi² par ensemble (fouilles récentes) ou par Unité Stratigraphique (fouilles Geneste et Chadelle 1982) et par catégorie de supports ou nucléus diagnostics. 1 : Pointes pseudo-Levallois ; 2 : Éclats débordants; 3 : Support retouché ; 4 : Nucléus de type Kombewa. En vert sont indiquées les $p$-value supérieure à un seuil de 0,05, en rouge sont indiquées les p-values inférieures à ce seuil.

Chi-square test per ensemble (recent excavation) or stratigraphic unit (Geneste and Chadelle excavations 1982) and per flake categories or diagnostic core. 1: Pseudo-Levallois points ; 2 : éclat débordant; 3 : Retouched blank; 4 : Kombewa-type core. P-values in green are greater than 0.05 cut off, those in red indicate $p$-values less than 0.05 .

\section{5 | DISCUSSION}

\section{1 | Des échantillons correspondant à la couche $H$ de D. Peyrony homogènes}

L'homogénéité des industries lithiques correspondant à la couche $\mathrm{H}$ de l'abri inférieur du Moustier peut être discutée (G\&C, Ensembles 1 et 2 G\&D).

Plusieurs arguments laissent entrevoir des échantillons homogènes :

- Tous les ensembles examinés $(\mathrm{H} 1+\mathrm{H} 2$; H4-H9 ; Ensemble 1; Ensemble 2) révèlent des chaînes opératoires Discoïdes exclusives dont l'objectif est la production de pointes pseudo-Levallois et d'éclats débordants.

- Ces chaînes opératoires sont ramifiées : des débitages de type Kombewa sont réalisés aux dépens des éclats corticaux sélectionnés pour leurs grandes dimensions et leur épaisseur importante, des éclats centrés et ou des éclats débordants sont produits au sein de séquences courtes.

- Les matières premières (principalement le Sénonien noir et blond) sont gérées de manière indifférenciée. Elles proviennent probablement des alluvions de la Vézère.

- Très peu de supports sont aménagés par retouche. Lorsqu'ils le sont, des couteaux à dos, des denticulés et des encoches sont aménagés. Contrairement aux éclats débordants et aux pointes pseudo-Levallois pour lesquels un bord actif est opposé à un dos constituant une partie préhensive, c'est l'existence d'un tranchant coupant opposé à un tranchant brut destiné à être aménagé après débitage qui définit le couteau à dos. L'intention d'aménager un dos intervient après le détachement du support. L'un de nous a précédemment décrit cette coexistence de couteaux à dos avec des éclats débordants et des pointes pseudo-Levallois comme formant un ensemble techno-fonctionnel cohérent, où la diversité des formes est probablement liée à des questions de préhension et d'usages (Gravina et Discamps 2015)

Cependant, il apparaît que les proportions de pointes pseudo-Levallois et d'éclats débordants ne sont pas homogènes au sein de la couche $H$. Sans évoluer drastiquement, ces proportions différentes révèlent selon nous une certaine flexibilité au sein des débitages de concept Discoïde puisqu'ils autorisent la production de ces deux types de supports dans des proportions variables sans pour autant dénaturer sa structure volumétrique.

\section{2 | Quelle place pour les ensembles 1 et 2 de l'abri inférieur du Moustier dans les archéoséquences du sud-ouest de la France?}

Les industries lithiques des ensembles 1 et 2 de la zone $B$, tout comme les sous-niveaux issus des fouilles G\&C correspondant à la couche H de D. Peyrony (Gravina et Discamps 2015) peuvent être attribués au technocomplexe lithique « Discoïde » (Faivre et al. 2017).

Le technocomplexe Discoïde regroupe des industries au sein desquelles la production d'éclats débordants et de pointes pseudo-Levallois est récurrente (Faivre et al. op. cit). La surface de débitage des nucléus Discoïdes ne demande pas d'entretien particulier, des axes de débitages cordaux assurent la mise en place de nervures guides et des convexités nécessaires à la production de ces éclats à la fois prédéterminant et prédéterminés. Les supports obtenus sont rarement retouchés et la ramification des chaînes opératoires est courante. Plusieurs assemblages partagent ces caractéristiques dans le bassin nord- 
aquitain dont les industries lithiques issues des sites de Combe-Grenal (c.14; Bourguignon et Turq 2003 ; Faivre et al. 2014), de Champs de Bossuet (Bourguignon et Turq 2003), des Fieux (c. I/J(s) ; K(s) ; Faivre 2006, Thiébaut 2005), du Régourdou (Faivre et al. 2016) et de Roc-de-Combe (c. Z2(s) ; Z2(q) ; Lorenzo Martinez et al. 2014). Au nordouest, s'ajoutent les industries lithiques issues des sites de Bois-Clair (Bernard-Guelle et al. 2017) et de SainteCésaire (couche Egpf; Thiébaut et al. 2009). Les industries lithiques des ensembles 1 et 2 de la zone B, tout comme celles des sous niveaux de la couches $\mathrm{H}$ issus des fouilles G\&C s'intègrent parfaitement à ce corpus de site où les débitages Discoïdes sont exclusifs.

D'après les chiffres évoqués par ces différents auteurs (fig. 11), la proportion de pointes pseudo-Levallois et d'éclats débordants varie sensiblement selon les études. Même si les critères de classifications inter-observateurs peuvent différer, cela démontre vraisemblablement une certaine variabilité dans les objectifs de débitage inhérents à ce concept ou à une forte mobilité de ces types de supports avec leur exportation hors des sites.

\section{3 | Existe-t-il une variabilité des objectifs des productions de type Kombewa?}

La ramification des chaînes opératoires est un phénomène bien connu dans les industries lithiques moustériennes (Bourguignon et al. 2004 ; Faivre 2008). Lorsque le débitage Discoïde est dominant dans les assemblages, la ramification des chaînes opératoires est très courante et met en évidence une certaine variabilité dans ses objectifs de production.

Sur le site de plein air de Champs de Bossuet (Bourguignon et Turq 2003) et aux Fieux (Faivre 2011), c'est la production de pointes pseudo-Levallois qui a guidé les chaînes opératoires de type Kombewa (fig.12). En revanche, dans la couche 14 de l'abri de Combe-Grenal, ce sont les éclats débordants et les éclats centrés qui dominent (Bourguignon et Turq 2003).

\section{Pointes Pseudo-Levallois}

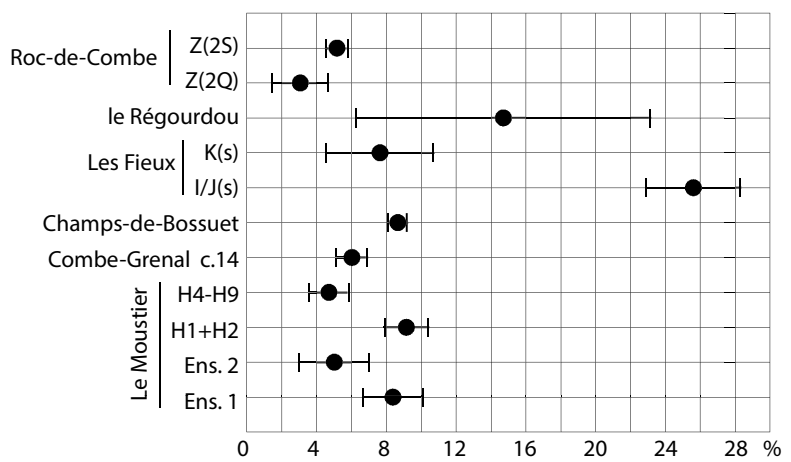

Si l'exportation des pointes pseudo-Levallois est parfois envisagée par ces auteurs, cette hypothèse n'est pas envisageable à l'abri inférieur du Moustier où l'observation des éclats-matrices confirment des chaînes opératoires très courtes au sein desquelles la recherche de pointes pseudo-Levallois n'est pas un objectif (cf. supra). Bien que les objectifs paraissent différents, le côté court (seulement quelques gestes) des chaînes opératoires de débitage sur éclats-matrices rapproche les industries de la couche 14 de Combe-Grenal et les ensembles archéo-stratigraphiques 1 et 2 de l'abri inférieur du Moustier.

Enfin, étant donné la disponibilité en matière première de bonne qualité dans l'environnement immédiat du site, la ramification des chaînes opératoires sur éclats-matrices ne semble pas liée à des contraintes d'ordre matériel.

\section{CONCLUSION}

La production des supports des ensembles 1 et 2 -en silex du Sénonien noirs et blonds probablement issus des alluvions locales de la Vézère et traités de manière indifférenciée- est effectuée aux dépens d'une chaîne opératoire Discoïde exclusive dont l'objectif est la production de pointes pseudo-Levallois et d'éclats débordants (Mourre 2003 ; Slimak 2003 ; Faivre et al. 2017). Cette chaîne opératoire est ramifiée. À partir de l'épaisseur des éclats corticaux produits en amont de la chaîne opératoire Discoïde, une modalité de débitage de type Kombewa est mise en place (Bourguignon et Turq 2003). Les tailleurs recherchent des produits débordants et des éclats centrés dont les tranchants semblent posséder des propriétés particulières. L'enchaînement des gestes lors des débitages de type Kombewa est réalisée au sein d'une séquence courte qui se limite à trois ou quatre enlèvements. Dans le cas général, il s'agit tout d'abord de l'ouverture d'un plan de frappe par une percussion donnée depuis la face inférieure de l'éclat-matrice. À partir de ce plan de frappe, un, deux ou trois éclats sont détachés sur la face inférieure de l'éclat-matrice.

\section{- FIGURE 11 -}

Comparaison des proportions de catégories diagnostics de supports par site avec intervalles de confiance à $95 \%$. Les données utilisées sont issues de cet article, mais aussi de Bourguignon et Turq 2003, Thiébaut 2005, Faivre 2006, Faivre et al. 2014, Lorenzo et al. 2014, Gravina et Discamps 2015, Faivre et al. 2016.
Comparison of the proportions of diagnostic technical categories by site with $95 \%$ confidence intervals. Data from Bourguignon and Turq 2003, Thiébaut 2005, Faivre 2006, Faivre et al. 2014, Lorenzo et al. 2014, Gravina and Discamps 2015, Faivre et al. 2017. 

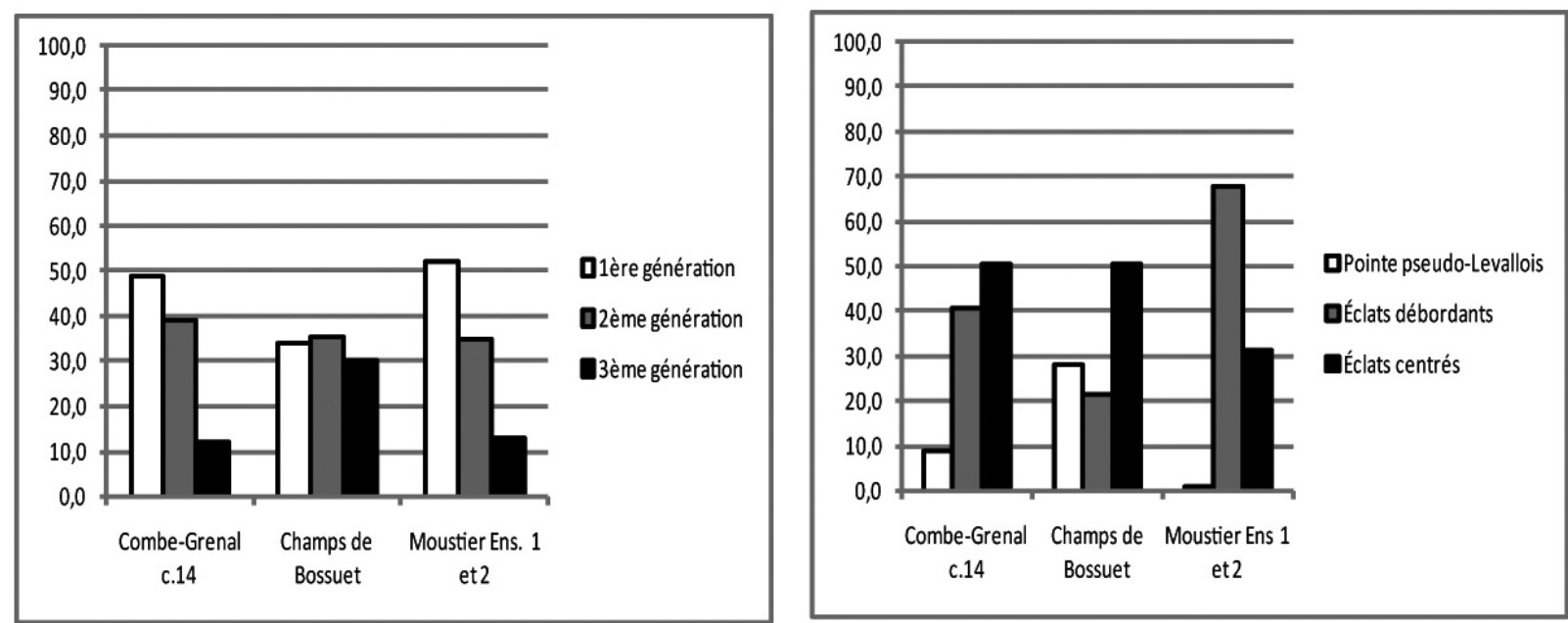

- FIGURE $12-$

à gauche : Histogramme des proportions de supports de type Kombewa par génération de Combe Grenal et Champs de Bossuet d'une part (Bourguignon et Turq 2003) et du Moustier (cette étude) d'autre part ; à droite : Histogramme des proportions de supports de type Kombewa par types de Combe Grenal et Champs de Bossuet d'une part (Bourguignon et Turq 2003) et du Moustier (cette étude) d'autre part.

Très peu de supports sont retouchés. Toutes les activités de taille sont réalisées in situ.

Les critères d'observation laissent entrevoir des ensembles de vestiges (G\&C, Ensembles 1 et 2) correspondant à la couche H de D. Peyrony homogènes du point de vue des industries lithiques, qu'il s'agisse des savoir-faire décrits et des connaissances impliquées (concept de débitage Discoïde et modalité de débitage de type Kombewa), des objectifs de production (recherche majoritaire de tranchants opposés à un dos) et des économies qui leur sont associées (débitage réalisé in situ, de manière indifférenciée selon le matériau).

\section{Ainsi,}

- Nos observations sur le matériel lithique issu des ensembles archéostatigraphiques 1 et 2 sont tout à fait comparables à celles effectuées sur la collection issue des fouilles J.-M. Geneste et J.-P. Chadelle correspondant également au sommet de la couche $\mathrm{H}$ de D. Peyrony (Gravina et Discamps, 2015). Elles laissent ainsi entrevoir une homogénéité de la production pour ces deux zones du site alors même qu'elles sont séparées de presque huit mètres.

- Les ensembles 1 et 2 peuvent être rattachés au technocomplexe « Discoïde » (Faivre et al. 2017). Alors que l'abri inférieur du Moustier était considéré comme un site clef dans l'invention (Bordes 1948 ; 1953) et la caractérisation (Soressi 2002) du technocomplexe lithique MTA de type B, cette attribution est ici une nouvelle fois remise en cause au profit du technocomplexe lithique Discoïde (Lorenzo Martinez et al., 2014 ; Gravina et Discamps 2015).

- La production d'éclats allongés est absente et les couteaux à dos, peu nombreux relativement aux pointes pseudo-Levallois et aux éclats débordants, sont aménagés
Right, proportions of Kombewa-type blanks by generation from Combe Grenal and Champs de Bossuet (Bourguignon and Tura 2003) and Le Moustier (this study).Left, proportions of Kombewa-type blanks by types from Combe Grenal and Champs de Bossuet (Bourguignon and Tura 2003) and Le Moustier (this study).

sur des éclats dont la face supérieure est partiellement corticale, probablement sélectionnés parmi les sousproduits des débitages Discoïdes.

- Les éléments exposés confirment que l'existence d'un MTA de type B à l'abri du Moustier est définitivement infirmée. Il en résulte que l'hypothèse d'une filiation entre le MTA de type B et le Châtelperronien perd fortement de la substance. (Gravina et Discamps 2015, cet article).

\section{REMERCIEMENTS}

Les nouvelles opérations de terrain au Moustier sont financées par la Direction Régionale des Affaires Culturelles Nouvelle Aquitaine et le Conseil Général de la Dordogne, que nous remercions pour leur soutien depuis 2014. Une partie des analyses rapportées ici a été réalisée dans le cadre d'un travail de Master à l'Université Toulouse Jean Jaurès et au sein de l'UMR5608 TRACES sous la direction de E. Discamps et B. Gravina. Ainsi, nous tenons à remercier l'équipe pédagogique de ce Master pour son soutien, ainsi que J.-G. Bordes et F. Bon, alors relecteurs de ce travail. Nous remercions J.-P. Faivre dont les conseils ont contribué à la révision de cet article. Enfin, les remarques et les recommandations de D. Hérisson et d'un deuxième relecteur sur une première version de ce manuscrit ont aidé à son amélioration.

\section{RÉFÉRENCES BIBLIOGRAPHIQUES}

BERNARD-GUELLE S., TAYLOR A., RUE M., FERNANDES P., AJAS A., COUDENNEAU A., HERNANDEZ M., MERCIER N. 2017 - Le site stratifié du Bois Clair à Montguyon (Charente-Maritime, France) : récurrences paléolithiques, brièveté des occupations et aires de débitage spécialisées, PALEO, n² 28, p. 41 
BOËDA E. 1994 - Le concept Levallois: variabilité des méthodes, CNRS éd., 280 p.

BOËDA E., GENESTE, J.-M., MEIGNEN, L. 1990 - Identification de chaînes opératoires lithiques du Paléolithique ancien et moyen. PALEO, $n^{\circ} 2$, p. 43-80.

BORDES F. 1948 - Les couches moustériennes du gisement du Moustier (Dordogne). Typologie et techniques de taille, Bulletin de la Société Préhistorique Française, tome 45, $n^{\circ}$ 4-5, p. 113-124.

BORDES F. 1953 - Essai de classification des industries « moustériennes », Bulletin de la Société préhistorique de France, tome 50, n 7-8, p. 457-466.

BORDES F. 1961 - Typologie du Paléolithique ancien et moyen, Delmas, Publications de l'Institut de Préhistoire de l'Université de Bordeaux, Mémoire n 1, Bordeaux, 111 p.

BOURGUIGNON L., FAIVRE J.-P., TURQ A. 2004 - Ramification des chaînes opératoires, une spécificité du moustérien?, PALEO, $n^{\circ} 16$, p 37-48

BOURGUIGNON L., TURQ A. 2003 - Une chaîne opératoire de débitage Discoïde sur éclat du Moustérien à denticulés aquitain : Les exemples de Champs de Bossuet et de Combe-Grenal c.14, In : Peresani, M. (éd.), Discoid Lithic Technology -Advances and implications, Oxford, BAR International Series 1120, 21p.

COLONGE D., MOURRE V. 2009 - Quartzite et quartzites : aspects pétrographiques, économiques et technologiques des matériaux majoritaires du Paléolithique ancien et moyen du sud-ouest de la France In : S. Grimaldi, S. Cura, (éd.), Technical analysis on quartzite exploitation, Oxford, BAR International Series 1998, p. 3-12.

DISCAMPS E., LEMEUR C. 2019 - Variabilité des proies chassées et modalités d'exploitation du Cerf au Moustérien : l'apport des collections récentes du Moustier (couches G et H), Paleo, $n^{\circ}$ 30, tome 1, p. 317-329.

DISCAMPS E., MUTH X., GRAVINA B., LACRAMPES-CUYAUBERES F., CHADELLE J.-P., FAIVRE J.-P., MAUREILLE B. 2016 Photogrammetry as a tool for interpreting archival data in archaeological fieldwork: Examples from the Middle Plaeolithic sites of Combe-Grenal, Le Moustier and Regourdou, Journal of Archaeological science: Reports, volume 8 , p. 268-276

FAIVRE J.-P. 2006 - L'industrie moustérienne du niveau Ks (locus 1) des Fieux (Miers, Lot) : mobilité humaine et diversité des compétences techniques, Bulletin de la Société préhistorique française, T. 103, p. 17-32

FAIVRE J.-P. 2008 - Organisation techno-économique des systèmes de production dans le Paléolithique moyen récent du Nord-Est aquitain : Combe-Grenal et Les Fieux. Thèse de doctorat, Université de Bordeaux I, Talence, 555 p.

FAIVRE J.-P. 2011 - Organisation techno-économique des systèmes de production dans le Paléolithique moyen récent du nord-est aquitain, Oxford, BAR International Series 2280, 244 p.
FAIVRE J.-P., DISCAMPS E., GRAVINA B., TURQ A., GUADELLI J.-L., LENOIR M. 2014 - The contribution of lithic production systems to the interpretation of Mousterian industrial variability in south-western France: the example of CombeGrenal (Dordogne, France). Quatern. Int. 350, p. 227-240.

FAIVRE J.-P., GRAVINA B., BOURGUIGNON L., DISCAMPS E., TURQ A. 2017 - Late Middle Palaeolithic technocomplexes (MIS 5-3) in the northeastern Aquitaine Basin: Advances and challenges, Quaternary International, $16 \mathrm{p}$.

GENESTE J.-M. 1985 - Analyse lithique d'industries moustériennes du Périgord: une approche technologique du comportement des groupes humains au Paléolithique moyen, thèse de doctorat, Université Bordeaux I, Bordeaux, $572 \mathrm{p}$.

GRAVINA B. 2017 - Intra-level technological change and its implications for Mousterian assemblage variability. The example of Le Moustier, layer G. Quaternary International 433, p. 132-139.

GRAVINA B., DISCAMPS E. 2015 - MTA-B or not to be? Recycled bifaces and shifting hunting strategies at Le Moustier and their implication for the late Middle Palaeolithic in southwestern France, Journal of Human Evolution, $n^{\circ}$ 84, p. 83-98.

HIGHAM T. et collaborateurs 2014 - The timing and spatiotemporal patterning of Neanderthal desappearance, Nature 512, p. 306-309

INIZAN M.-L., REDURON M., ROCHE H., TIXIER J. 1995 Technologie de la pierre taillée, tome 4, Meudon, C.R.E.P., $199 \mathrm{p}$.

JAUBERT J. 2011 - Les archéoséquences du Paléolithique moyen du Sud-Ouest de la France : quel bilan un quart de siècle après François Bordes ? In : F. Delpech et J. Jaubert (dir.), François Bordes et la Préhistoire, Colloque international François Bordes, Bordeaux 22-24 avril 2009, Paris, éd. du CTHS, p. 235-253.

LARTET E., CHRISTY H. 1865-1875 - Reliquiae Aquitanicae, being contributions to the archaeology and Palaeontology of Perigord and the adjoining provinces of Southern France, Londres, $506 \mathrm{p}$.

LAVILLE H., RIGAUD, J.-P. 1973 - L'abri inferieur du Moustier (Dordogne). Précisions stratigraphiques et chronologiques. C. R. Acad. Sci. Paris 276, p. 3097-3100.

LOCHT J.-L., SWINNEN C. ANTOINE P., AUGUSTE P., PATOUMATHIS M., DEPAEPE P., FALGUERES C., LAURENT M., BAHAIN J.-J., MATHYS P. 1995 - Le gisement paléolithique moyen de Beauvais (Oise), Bulletin de la Société Préhistorique Française, T. 92, n² 2, p. 213-226.

LORENZO MARTINEZ M., BORDES J.-G., JAUBERT J. 2014 L'industrie lithique du Paléolithique moyen récent de Roc de Combe (Payrignac, Lot, France), un nouvel exemple de Moustérien Discoïde à denticulés, PALEO, n² 25, p. 101-124.

MELLARS P. 1996 - The Neanderthal legacy: an archaeological perspective from Western Europe, Princeton University Press, Princeton 
MELLARS P., GRUN 1991 - A comparison of the Electron Spin Resonance and thermoluminescence dating methods : the results of ESR dating at le Moustier (France), Cambridge Archaeological Journal, 1(2), p. 269-276

MORTILLET (de) G. 1869 - Essai d'une classification des cavernes et des stations sous abri fondée sur les produits de l'industrie humaine, Typographie de Bonnal et Gibrac, 8 p.

MOURRE V. 2003 - Discoïde ou pas Discoïde ? Réflexions sur la pertinence des critères techniques définissant le débitage Discoïde, In : Peresani, M. (éd.), Discoid Lithic Technology -Advances and implications, Oxford, BAR International Series1120, 18 p.

PELEGRIN J, KARLIN C, BODU P. 1988 - «chaînes opératoires» : un outil pour le préhistorien, Technologie préhistorique, Notes et Monographies techniques 25, CNRS, Paris, p. 55-62.

PERESANI M. (éd.) 2003 - Discoid lithic technology : Advances and inplications, Oxford, BAR International Series 1120, 288 p.

PERLĖS C. 1991 - Économie des matières premières et économie du débitage : deux conceptions opposées ?, 25 ans d'Études technologiques en Préhistoire, XIe Rencontres Internationales d'Archéologie et d'Histoire d'Antibes, ADPCA, Juan les-Pins, p. 35-45.

PEYRONY D. 1920 - Le Moustérien - ses faciès., Association Française pour l'avancement des Sciences, $44^{\circ}$ session, Strasbourg, p. 1-2.

PEYRONY D. 1930 - Le Moustier. Ses gisements, ses industries, ses couches géologiques. Revue Anthropologique, tome XL, p. 48-76 et p. 155-176.

PROST D.-C. 1989 - Enlèvements accidentels, enlèvements d'utilisation et de retouche sur les outils de pierre taillée, Université de Paris X - Nanterre, thèse de doctorat, 552 p.

RIVIĖRE É. 1908 - Le squelette humain chélléo-moustérien du Moustier-de-Peyzac (Dordogne), Bulletin de la Société Préhistorique de France, tome 5, nº 9, p. 441-442.

RIVIĖRE É. 1909 - De l'antiquité paléolithique du squelette humain du Moustier-de-Peyzac (Dordogne), Bulletin de la société préhistorique de France, tome 6, n³, p. 142-144.

RUEBENS K. 2013 - Regional behaviour among the late Neanderthal groups in Western Europe: a comparative assessment of late Middle Palaeolithic bifacial tool variability, Journal of Human Evolution, Volume 65, Issue 4, p. 341-362.

SLIMAK L. 2003 - Les débitages Discoïdes moustériens: évaluation d'un concept technologique in M. Peresani (éd.), Discoid Lithic Technology - Advances and implications, Oxford, BAR International Series 1120, p. 1-18.

SONNEVILLE-BORDES (de) D. 1960 - Le paléolithique supérieur en Périgord, Delmas, Bordeaux, 2 vol. 558 p.

SORESSI M. 1999 - Variabilité technologique au Moustérien. Analyse comparée du débitage Levallois MTA A du Moustier (Dordogne, France), PALEO, nº11, p. 111-134.
SORESSI M. 2002 - Le Moustérien de tradition acheuléenne du sud-ouest de la France, Discussion sur la signification des faciès à partir de l'étude comparée de 4 sites: Pech de l'Azé I, le Moustier, La Rochette et la Grotte XVI, thèse de doctorat, Université de Bordeaux I, Bordeaux, 344 p.

SORESSI M., ROUSSEL M. 2014 - European Middle to Upper Palaeolithic Transitional Industries: Châtelperronian, In: Smith, C. (éd.), Encyclopedia of Global Archaeology. Springer, New-York, p. 2679-2693.

STUDENT 1908- The Probable Error of a Mean, Biometrika, vol.6, $\mathrm{n}^{\circ} 1$, p. 1-25.

TAVOSO A. 1978 - Le paléolithique inférieur et moyen du Haut-Languedoc: (gisements des terrasses alluviales du Tarn, du Dadou, de l'Agout, du Sor et du Fresquel), Institut de Paléontologie Humaine, Paris, Études quaternaires 5, $404 \mathrm{p}$.

THIÉBAUT C. 2005 - Le Moustérien à denticulés : variabilité ou diversité technoéconomique?, Université de Provence, Aix-en-Provence, thèse de doctorat, 2 vol., 870 p.

THIÉBAUT C., MEIGNEN L., LEVEQUE F. 2009 - Les dernières occupations moustéri- ennes de Saint-Césaire (CharenteMaritime, France). Bulletin de la Société Préhistorique Française, T. 106, p. 691-714.

THOMAS M. 2017 - Taphonomie et techno-économie de palimpsestes d'occupations : l'exemple de la couche $\mathrm{H} d u$ Moustier (Saint-Léon sur Vézère, Dordogne), Université Toulouse Jean Jaurès, Mémoire de Master 2, 69 p.

THOMAS M., DISCAMPS E., GRAVINA B., TEXIER J.-P. 2019 Analyse taphonomique et spatiale de palimpsestes d'occupations moustériennes de l'abri inférieur du Moustier (Dordogne, France), Paleo, n³0, tome 1, p. 278-299.

TIXIER J., INIZAN M.-L., ROCHE H. 1980 - Préhistoire de la pierre taillée 1, terminologie et technologie. In : Cercle de Recherches et d'Études Préhistoriques. Antibes, 120 p.

TIXIER J., TURQ A. 1999 - Kombewa et alii, PALEO, n 11, p. 135-143.

TURQ A., FAIVRE J.-P., GRAVINA B., BOURGUIGNON L. 2016 Building models of Neanderthal territories from raw material transport in the Aquitaine basin (southwestern France), Quaternary International 443, p. 88-101.

TURQ A., MORALA A., SÉRONIE-VIVIEN M.-R. 2013 - Inventaire des silicifications du Quercy, de ses marges et des marqueurs lithologiques du nord-est aquitain: synthèse des données. In : Jarry, M., Brugal, J.-P., Ferrier, C. (dir.), Modalité d'occupation et d'exploitation des milieux dans le sud Ouest de la France: l'exemple du Quercy, PALEO, supplément $n^{\circ}$ 4. p. 159-180.

VALLADAS H., GENESTE J.-M., JORON J.-M., CHADELLE J.-P. 1986 - Thermoluminescence dating of the Moustier (Dordogne, France), Nature, $n^{\circ} 322$, p. 452-454. 